\title{
Systematic derivation of a surface polarisation model for planar perovskite solar cells $\dagger$
}

\author{
N. E. COURTIER ${ }^{1}$, J. M. FOSTER ${ }^{2}$, S. E. J. O’KANE ${ }^{3}$, \\ A. B. WALKER ${ }^{3}$ and G. RICHARDSON ${ }^{1}$ \\ ${ }^{1}$ Mathematical Sciences, University of Southampton, SO17 1BJ, UK \\ emails:nc4g14@soton.ac.uk, G.Richardson@soton.ac.uk \\ ${ }^{2}$ Department of Mathematics, University of Portsmouth, PO1 $3 H F, U K$ \\ email: jamie.michael.foster@gmail.com \\ ${ }^{3}$ Department of Physics, University of Bath, BA2 7AY, UK \\ emails: A.B.Walker@bath.ac.uk, S.E.J.O'Kane@bath.ac.uk
}

(Received 7 December 2017; revised 19 March 2018; accepted 24 March 2018; first published online 22 April 2018)

\begin{abstract}
Increasing evidence suggests that the presence of mobile ions in perovskite solar cells (PSCs) can cause a current-voltage curve hysteresis. Steady state and transient current-voltage characteristics of a planar metal halide $\mathrm{CH}_{3} \mathrm{NH}_{3} \mathrm{PbI}_{3} \mathrm{PSC}$ are analysed with a drift-diffusion model that accounts for both charge transport and ion vacancy motion. The high ion vacancy density within the perovskite layer gives rise to narrow Debye layers (typical width $\sim 2 \mathrm{~nm}$ ), adjacent to the interfaces with the transport layers, over which large drops in the electric potential occur and in which significant charge is stored. Large disparities between (I) the width of the Debye layers and that of the perovskite layer $(\sim 600 \mathrm{~nm})$ and (II) the ion vacancy density and the charge carrier densities motivate an asymptotic approach to solving the model, while the stiffness of the equations renders standard solution methods unreliable. We derive a simplified surface polarisation model in which the slow ion dynamics are replaced by interfacial (non-linear) capacitances at the perovskite interfaces. Favourable comparison is made between the results of the asymptotic approach and numerical solutions for a realistic cell over a wide range of operating conditions of practical interest.
\end{abstract}

Key words: perovskite, solar cell, ion vacancy, drift-diffusion, asymptotic analysis.

\section{Introduction}

Since the first use of methylammonium lead tri-halide perovskite as a sensitizer in a dye-sensitized solar cell [15], and its subsequent incorporation into a novel thin film solar technology as a bulk solar absorber [14,17], the efficiency of perovskite solar cells (PSCs) has increased extremely rapidly from around 3\% to above $20 \%$ [6], a level that is comparable to the standard crystalline silicon devices. This increase, along with advances

$\dagger$ NEC is supported by an EPSRC funded studentship from the CDT in New and Sustainable Photovoltaics. SEJO'K was supported by EPSRC grant EP/J017361/1. ABW acknowledges funding from the European Union Horizon 2020 research and innovation programme under Grant no. 676629 . 

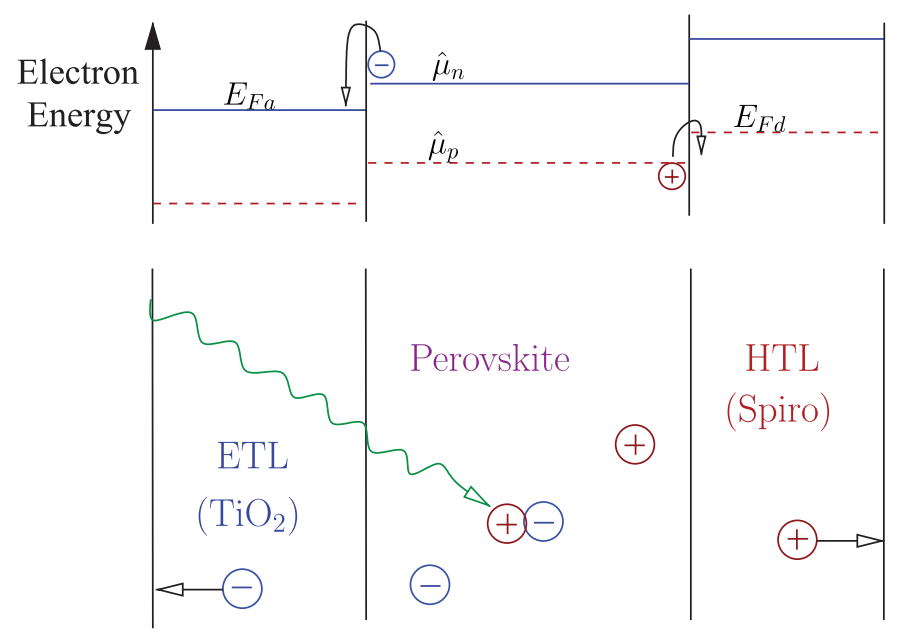

FIGURE 1. (Top) Band diagram showing that holes preferentially move from the perovskite to the HTL and electrons to the ETL. (Bottom) Schematic of a planar PSC showing photogeneration and transport of electrons and holes.

in the material properties and stability of PSCs, makes this area of photovoltaic research a very hot topic [22,38].

Typically, PSCs contain a three-layer architecture consisting of a layer of semiconducting perovskite absorber sandwiched between a semiconducting hole-transport layer (HTL) and a semiconducting electron-transport layer (ETL), see Figure 1. These transport layers are also referred to as selective or extraction layers or, alternatively, electron- and hole-blocking layers. A common pair of hole- and electron-transport materials are SpiroMeOTAD (2,2'7,7'-tetrakis-(N,N-di-p-methoxyphenyl amine)-9,9'-spirobifluorene, here referred to as spiro) and titanium dioxide $\left(\mathrm{TiO}_{2}\right)$, respectively. Absorption of light occurs predominantly within the perovskite layer and is associated with the generation of an exciton which, due to its weak binding energy $(\sim 50 \mathrm{meV})[16]$, rapidly dissociates into a free electron in the conduction band, and a hole in the valence band, of the perovskite. These charge carriers move both in response to random thermal excitations (diffusion) and to internal electric fields (drift). The hole- and electron-transport materials are chosen such that their band energies give rise to a built-in electric field across the perovskite that separates the charge carriers. The field drives holes towards the HTL and electrons towards the ETL, generating a current at biases between zero and open circuit. Furthermore, the conduction band energy in the HTL is significantly above that in the perovskite, so that a potential barrier exists to the entry of electrons into this material from the perovskite. Similarly, the valence band energy in the ETL is significantly below that in the perovskite, so that a potential barrier exists to the entry of holes into this material from the perovskite.

An unusual feature of PSCs is their long timescale transient behaviour occurring on the order of tens of seconds. This behaviour is exemplified by so-called current-voltage hysteresis [35] whereby apparent hysteresis loops are observed in current-voltage $(J-V)$ curves obtained by sweeping the voltage across a cell, from high to low and back again, 
and measuring the current as a function of voltage. From a practical point of view, this hysteresis has led to some unfortunate consequences, including inflated reports of power conversion efficiencies (PCEs) given that PCEs are often calculated from a currentvoltage sweep. Long timescale transient behaviour has also been observed in dark current transients (whereby the cell is first held in the dark, then the applied voltage is suddenly changed and the resulting current measured) [23]. More recently, very long timescale transients lasting many hours have been observed in cell efficiency [9]. These decays in PCE can be reversed by allowing the cell to recover in the dark. Various explanations have been proposed for these transient behaviours, including (a) large trap state densities close to the interfaces with the transport layers, (b) slow ferroelectric polarisation of the perovskite material and (c) the motion of iodide $\left(\mathrm{I}^{-}\right)$vacancies within the perovskite material [35]. As discussed in Richardson et al. [28], it is now widely accepted that the only one of these mechanisms capable of explaining the data is iodide vacancy motion.

Various approaches may be used to model PSCs ranging from atomistic density functional theory (DFT) simulations, to drift-diffusion models of charge carrier and ion motion, to lumped parameter device models (equivalent circuits). DFT calculations, while perhaps the most fundamental approach, are so computationally intensive that they are incapable of describing the behaviour of a full cell. In practice, they are used to obtain estimates of macroscopic quantities, such as ion vacancy densities and mobilities, from the atomistic structures of the materials forming the device [10]. In contrast, drift-diffusion models, which are applicable on the nanometre length scale and upward, describe the motion of electrons, holes and ion vacancies. Such models have been presented and solved in a number of works $[5,9,13,21,23,28,30,34,40]$. However, it is notable that, with the exception of two [23,28], all of these works use parameter values that are very far from realistic. This may be ascribed to the extreme numerical stiffness of the problem owing to very narrow $(\sim 2 \mathrm{~nm})$ Debye (boundary) layers that form as a result of ion accumulation/depletion at the edges of the perovskite layer. In order to overcome this difficulty, Richardson et al. [28] adopted a combined numerical and asymptotic approach, in which the electrical properties of the Debye layers are modelled by a non-linear surface (Debye layer) capacitance, based on estimates for the equilibrium ion vacancy density and mobility obtained from DFT calculations performed by Eames et al. [10]. The purpose of that work was to demonstrate that experimental $J-V$ hysteresis data could be explained by the motion of ion vacancies in the perovskite layer and so the derivation of the asymptotic solution was not given there.

The aim of this paper is to systematically derive the asymptotic approach used in the earlier work by Richardson et al. [28] and validate it against numerical solutions to the full model. A similar approach has been used for (i) asymptotic derivations of equivalent circuit models from drift-diffusion models [11,12,32] (in the context of organic solar cells, PSCs and bipolar silicon devices, respectively); (ii) a matched asymptotic analysis of np-diodes [24]; (iii) asymptotic derivations of the standard 'regional' models of semiconductors from a drift-diffusion model [31]; (iv) multidimensional models of bulk heterojunction solar cells $[3,29]$; and (v) the asymptotic analysis of quantum drift-diffusion models [2]. Subsequent to Richardson et al. [28], Ravishankar et al. [26] published a heuristic model similar to the surface capacitance model used in this earlier work, which 
they term a surface polarisation model. We argue that the systematic derivation of such models from the underlying drift-diffusion equations, as here, has the significant advantage of directly relating the surface capacitances to the device physics.

This work is set out as follows. In Section 2, we formulate the drift-diffusion model for a PSC, non-dimensionalise and estimate the model parameters. In Section 3, we use formal asymptotic methods, based on the parameter estimates made in Section 2, to derive a hierarchy of simplified models to the full PSC model including the surface polarisation model of Ravishankar et al. [26]. In Section 4, the results of the simplified models are compared to numerical solutions of the full PSC model and finally, in Section 5, we draw our conclusions.

\section{Problem formulation}

Here, we consider a perovskite absorber layer, sandwiched between an ETL and an HTL (typically $\mathrm{TiO}_{2}$ and spiro, respectively). We make the assumption that the transport layers are sufficiently highly doped that they are effectively equipotential across their width and take the same potential as their respective contacts. In the perovskite, in line with DFT calculations on its chemical structure [10], we assume there exists a high density of mobile anion vacancies, in addition to the charge carriers. The resulting dimensional model for the perovskite layer $(0<x<b)$, following earlier work [28], is outlined below.

\section{Dimensional model}

Conservation of holes (density $p$ ) and conduction electrons (density $n$ ) is described by

$$
\begin{aligned}
& \frac{\partial p}{\partial t}+\frac{1}{q} \frac{\partial j_{p}}{\partial x}=G-R, \quad j_{p}=-q D_{p}\left(\frac{\partial p}{\partial x}+\frac{p}{V_{T}} \frac{\partial \phi}{\partial x}\right), \\
& \frac{\partial n}{\partial t}-\frac{1}{q} \frac{\partial j_{n}}{\partial x}=G-R, \quad j_{n}=q D_{n}\left(\frac{\partial n}{\partial x}-\frac{n}{V_{T}} \frac{\partial \phi}{\partial x}\right),
\end{aligned}
$$

where $G$ is the photo-generation rate; $R(n, p)$ is the bulk recombination and thermal generation rate (henceforth abbreviated to recombination rate); $\phi$ is the electric potential; $j_{n}$ and $j_{p}$ are electron- and hole-currents, respectively; and $V_{T}=k T / q$ is the thermal voltage. Similar equations for the conservation of positively-charged anion vacancies (density $P$ ) and negatively charged cation vacancies (density $N$ ) take the form

$$
\begin{aligned}
\frac{\partial P}{\partial t}+\frac{\partial \mathcal{F}_{p}}{\partial x}=0, & \mathcal{F}_{p}=-D_{+}\left(\frac{\partial P}{\partial x}+\frac{P}{V_{T}} \frac{\partial \phi}{\partial x}\right), \\
\frac{\partial N}{\partial t}+\frac{\partial \mathcal{F}_{n}}{\partial x}=0, & \mathcal{F}_{n}=-D_{-}\left(\frac{\partial N}{\partial x}-\frac{N}{V_{T}} \frac{\partial \phi}{\partial x}\right),
\end{aligned}
$$

where $\mathcal{F}_{p}$ (and $\mathcal{F}_{n}$ ) are the fluxes of the positive (and negative) ion vacancies (as opposed to the current fractions carried by these species). Both sets of equations couple to Poisson's equation for the electric potential

$$
\frac{\partial^{2} \phi}{\partial x^{2}}=\frac{q}{\varepsilon}(N-P+n-p) .
$$


Boundary conditions at the edges of the perovskite, $x=0$ (the interface with the ETL) and $x=b$ (the interface with the HTL) take the form

$$
\left.\left.\begin{array}{c}
n=n_{0} \\
\phi=\frac{V_{b i}-V_{a p}}{2} \\
j_{p}=-q R_{l} \\
\mathcal{F}_{n}=0 \\
\mathcal{F}_{p}=0
\end{array}\right\} x=0, \quad \begin{array}{c}
p=p_{0} \\
\\
j_{n}=-q R_{r} \\
\mathcal{F}_{n}=0 \\
\mathcal{F}_{p}=0
\end{array}\right\} x=b
$$

where $V_{a p}$ is the applied voltage; $V_{b i}$ is the built-in potential; $R_{l}$ and $R_{r}$ are the interfacial charge recombination rates on $x=0$ and $x=b$, respectively; and the carrier densities on the interfaces are given by the expressions (see e.g., Nelson [20])

$$
p_{0}=g_{v} \exp \left(\frac{\hat{\mu}_{p}-E_{F_{d}}}{k T}\right), \quad n_{0}=g_{c} \exp \left(\frac{E_{F_{a}}-\hat{\mu}_{n}}{k T}\right) .
$$

Here, $g_{c}$ and $g_{v}$ are the effective density of states in the conduction and valence bands of the perovskite, respectively; $\hat{\mu}_{n}$ and $\hat{\mu}_{p}$ are the perovskite conduction and valence band energies, respectively. In addition, we model the highly doped ETL and HTL as metals in which $E_{F_{d}}$, the HOMO energy level of the HTL (Spiro), and $E_{F_{a}}$, the conduction band energy of the ETL $\left(\mathrm{TiO}_{2}\right)$, play the roles of the Fermi levels in these materials. These equations are supplemented by initial conditions, which we choose as follows to ensure charge neutrality,

$$
\left.p\right|_{t=0}=p_{0},\left.\quad n\right|_{t=0}=n_{0},\left.\quad N\right|_{t=0}=N_{0},\left.\quad P\right|_{t=0}=N_{0} .
$$

\section{The built-in voltage}

This quantity can be found from (2.1) with boundary conditions (2.4) by noting that, at equilibrium, the photo-generation rate, applied voltage and electron- and hole-currents are all zero $\left(G=0, V_{a p}=0\right.$ and $\left.j_{p}=j_{n}=0\right)$. The equilibrium solutions for $n$ and $p$ have the form

$$
p=A \exp \left(-\frac{\phi}{V_{T}}\right), \quad n=B \exp \left(\frac{\phi}{V_{T}}\right),
$$

in which the constants $A$ and $B$ are determined by the boundary conditions such that

$$
p=p_{0} \exp \left(-\frac{\phi}{V_{T}}-\frac{V_{b i}}{2 V_{T}}\right), \quad n=n_{0} \exp \left(\frac{\phi}{V_{T}}-\frac{V_{b i}}{2 V_{T}}\right) .
$$

Furthermore, since the rate of thermal generation and recombination must be equal $(R=0)$ at equilibrium (see e.g., (2.7)), we require $n p=n_{i}^{2}$. It follows that

$$
V_{b i}=V_{T} \log \left(\frac{n_{0} p_{0}}{n_{i}^{2}}\right)
$$

which with the parameter estimates in Table 1 , turns out to be $1 \mathrm{~V} \approx 39 V_{T}$. 
Table 1. Parameters for the device described in Section 2.2, where $\varepsilon_{0}$ is the permittivity of free space and DoS is an abbreviation for density of states. Here, $\alpha$ is calculated from Loper et al. [18] based on light wavelength of $585 \mathrm{~nm}$ (close to the peak absorption of the perovskite layer). Unless stated otherwise, the parameters are for the perovskite layer.

\begin{tabular}{llll}
\hline \hline Symbol & \multicolumn{1}{c}{ Description } & \multicolumn{1}{c}{ Value } & Source \\
\hline$T$ & Temperature & $298 \mathrm{~K}$ & \\
$F_{p h}$ & Incident photon flux & $9.5 \times 10^{20} \mathrm{~m}^{-2} \mathrm{~s}^{-1}$ & {$[18,28]$} \\
$\alpha$ & Absorption coefficient & $6.1 \times 10^{6} \mathrm{~m}^{-1}$ & {$[18]$} \\
$b$ & Width & $1.5-6 \times 10^{-7} \mathrm{~m}$ & {$[17,25]$} \\
$V_{b i}$ & Built-in voltage & $1 \mathrm{~V}$ & \\
$\hat{\mu}_{n}$ & Conduction band level & $-3.7 \mathrm{eV}$ & {$[33]$} \\
$\hat{g}_{c}$ & Conduction band DoS & $8.1 \times 10^{24} \mathrm{~m}^{-3}$ & {$[4]$} \\
$\hat{g}_{v}$ & Valence band DoS & $5.8 \times 10^{24} \mathrm{~m}^{-3}$ & {$[4]$} \\
$\hat{\mu}_{p}$ & Valence band level & $-5.4 \mathrm{eV}^{-4}$ & {$[33]$} \\
$D_{n}$ & Electron diffusion coefficient & $1.7 \times 10^{-4} \mathrm{~m}^{2} \mathrm{~s}^{-1}$ & {$[36]$} \\
$D_{p}$ & Hole diffusion coefficient & $1.7 \times 10^{-4} \mathrm{~m}^{2} \mathrm{~s}^{-1}$ & {$[36]$} \\
$D_{+}$ & Vacancy diffusion coefficient & $2.4 \times 10^{-16} \mathrm{~m}^{2} \mathrm{~s}^{-1}$ & {$[10,28]$} \\
$N_{0}$ & Vacancy density & $1.6 \times 10^{19} \mathrm{~cm}^{-3}$ & {$[41]$} \\
$\varepsilon_{p}$ & Permittivity & $24.1 \varepsilon_{0}$ & {$[4]$} \\
$\tau_{n}$ & Electron pseudo-lifetime & $3 \times 10^{-12} \mathrm{~s}^{-10}$ & {$[28]$} \\
$\tau_{p}$ & Hole pseudo-lifetime & $9 \times 10^{-10} \mathrm{~s}^{-3}$ & {$[28]$} \\
$g_{c}$ & TiO ${ }_{2}$ conduction band DoS & $8.1 \times 10^{24} \mathrm{~m}^{-3}$ & {$[4]$} \\
$g_{v}$ & Spiro valence band DoS & $5.8 \times 10^{24} \mathrm{~m}^{-3}$ & {$[4]$} \\
$E_{F a}$ & TiO Fermi level & $-4.0 \mathrm{eV}$ & {$[33]$} \\
$E_{F d}$ & Spiro Fermi level & $-5.0 \mathrm{eV}$ & {$[33]$} \\
\hline \hline
\end{tabular}

\section{Recombination and photo-generation}

At the radiation intensities associated with sunlight, the bulk recombination rate within the perovskite, $R$, is believed to be predominantly trap assisted (although at higher radiation intensities bimolecular recombination becomes significant) [37]. It is therefore appropriate to model bulk recombination by the Shockley-Read-Hall rate equation (see e.g., Nelson [20] Section 4.5.5)

$$
R=\frac{n p-n_{i}^{2}}{\tau_{p} n+\tau_{n} p+k_{3}},
$$

where $\tau_{n}$ and $\tau_{p}$ are the pseudo-lifetimes of conduction electrons and holes, respectively, and $k_{3}$ is a constant related to the pseudo-lifetimes and trap state energy level (typically negligible to the other terms in the denominator of (2.7) when the cell is under illumination). Furthermore, Stranks et al. [37] suggest that bulk recombination is hole dominated $\left(\tau_{p} \gg \tau_{n}\right)$, an assumption which is in line with that made in Richardson et al. [28]. There is still no consensus on the relative importance of interfacial recombination (at the interfaces between the perovskite and the transport layers) in comparison to bulk recombination although we note that this may be sample dependent. For example, de Quilettes et al. [8] note that recombination within the perovskite occurs predominantly 
at crystal boundaries, which implies the magnitude of bulk recombination is strongly dependent upon the perovskite structure.

The photo-generation rate, $G$, is assumed to follow the Beer-Lambert law of light absorption; with light entering the device through the ETL $\left(\mathrm{TiO}_{2}\right)$. This has the form

$$
G=F_{p h} \alpha \exp (-\alpha x)
$$

where $F_{p h}$ is the incident photon flux and $\alpha$ is the light absorption coefficient of the perovskite.

\subsection{Non-dimensionalisation}

Dimensionless variables (denoted by a star) are introduced by rescaling (i) space with the width of the perovskite layer; (ii) voltages with the thermal voltage; (iii) charge carrier densities with the typical photo-generated charge density, $\Pi_{0}$ (see (2.10)); (iv) current densities with the typical photo-generated current density, $q F_{p h}$; and (v) ion densities with the typical ion density, $N_{0}$. The rescaling reads

$$
\begin{array}{lll}
x=b x^{*}, & \phi=V_{T} \phi^{*}, & V_{a p}=V_{T} \Phi^{*}, \\
t=\tau_{\text {ion }} t^{*}, & p=\Pi_{0} p^{*}, & n=\Pi_{0} n^{*}, \\
j_{p}=q F_{p h} j_{p}{ }^{*}, & j_{n}=q F_{p h} j_{n}{ }^{*}, & P=N_{0} P^{*}, \\
N=N_{0} N^{*}, & \mathcal{F}_{p}=\frac{D_{+} N_{0}}{b} \mathcal{F}_{p}^{*}, & \mathcal{F}_{n}=\frac{D_{+} N_{0}}{b} \mathcal{F}_{n}^{*}, \\
G=\frac{F_{p h}}{b} G^{*}, & R=\frac{F_{p h}}{b} R^{*}, & R_{l, r}=F_{p h} R_{l, r}^{*} .
\end{array}
$$

Here, $L_{d}$ is the Debye length calculated on the basis of the ion vacancy density and $\tau_{\text {ion }}$ is the characteristic timescale for ion motion defined, respectively, by

$$
L_{d}=\left(\frac{\varepsilon V_{T}}{q N_{0}}\right)^{1 / 2}, \quad \tau_{i o n}=\frac{L_{D} b}{D_{+}} .
$$

Furthermore, we take $\Pi_{0}$ to be the characteristic charge carrier density required to remove the photo-generated charge in the absence of an electric field

$$
\Pi_{0}=\frac{F_{p h} b}{\hat{D}},
$$

where $\hat{D}$ is a typical carrier diffusivity. The non-dimensionalisation gives rise to the following dimensionless quantities that characterise the system:

$$
\begin{array}{lllll}
v=\frac{D_{+} b}{\hat{D} L_{d}}, & \kappa_{p}=\frac{D_{p}}{\hat{D}}, & \kappa_{n}=\frac{D_{n}}{\hat{D}}, & \bar{n}=\frac{n_{0}}{\Pi_{0}}, & \bar{p}=\frac{p_{0}}{\Pi_{0}}, \\
\gamma=\frac{b^{2}}{\hat{D} \tau_{p}}, & \Delta=\frac{D_{-}}{D_{+}}, & N_{i}=\frac{n_{i}}{\Pi_{0}}, & \lambda=\frac{L_{d}}{b}, & \delta=\frac{\Pi_{0}}{N_{0}}, \\
\Phi_{b i}=\frac{V_{b i}}{V_{T}}, & \Upsilon=\alpha b, & \epsilon=\frac{\tau_{n}}{\tau_{p}}, & K_{3}=\frac{k_{3}}{\Pi_{0} \tau_{p}} .
\end{array}
$$




\section{The dimensionless problem}

The system of equations obtained by applying the rescaling (2.9) to the variables in $(2.1)-(2.8)$ is

$$
\left.\left.\begin{array}{cc}
v \frac{\partial p^{*}}{\partial t^{*}}+\frac{\partial j_{p}^{*}}{\partial x^{*}}=G^{*}-R^{*}, & j_{p}^{*}=-\kappa_{p}\left(\frac{\partial p^{*}}{\partial x^{*}}+p^{*} \frac{\partial \phi^{*}}{\partial x^{*}}\right), \\
v \frac{\partial n^{*}}{\partial t^{*}}-\frac{\partial j_{n}^{*}}{\partial x^{*}}=G^{*}-R^{*}, & j_{n}^{*}=\kappa_{n}\left(\frac{\partial n^{*}}{\partial x^{*}}-n^{*} \frac{\partial \phi^{*}}{\partial x^{*}}\right), \\
\frac{\partial P^{*}}{\partial t^{*}}+\lambda \frac{\partial \mathcal{F}_{p}^{*}}{\partial x^{*}}=0, & \mathcal{F}_{p}^{*}=-\left(\frac{\partial P^{*}}{\partial x^{*}}+P^{*} \frac{\partial \phi^{*}}{\partial x^{*}}\right), \\
\frac{\partial N^{*}}{\partial t^{*}}+\lambda \frac{\partial \mathcal{F}_{n}^{*}}{\partial x^{*}}=0, & \mathcal{F}_{n}^{*}=-\Delta\left(\frac{\partial N^{*}}{\partial x^{*}}-N^{*} \frac{\partial \phi^{*}}{\partial x^{*}}\right), \\
\left.\frac{\partial^{2} \phi^{*}}{\partial x^{* 2}}=\frac{1}{\lambda^{2}}\left[N^{*}-P^{*}+n^{*}-p^{*}\right)\right] \\
n^{*}=\frac{\Phi_{b i}-\Phi^{*}}{2} \\
j_{p}^{*}=-q R_{l}^{*} \\
\mathcal{F}_{n}^{*}=0 \\
\mathcal{F}_{p}^{*}=0
\end{array}\right\} \begin{array}{c}
p^{*}=\bar{p} \\
x^{*}=0, \\
p^{*}=-\frac{\Phi_{b i}-\Phi^{*}}{2} \\
j_{n}^{*}=-q R_{r}^{*} \\
\mathcal{F}_{n}^{*}=0 \\
\mathcal{F}_{p}^{*}=0 \\
N^{*}=1, \\
P^{*}=1
\end{array}\right\}
$$

The dimensionless recombination and generation rates (for a device under constant illumination) are given by

$$
R^{*}\left(n^{*}, p^{*}\right)=\gamma\left(\frac{n^{*} p^{*}-N_{i}^{2}}{n^{*}+\epsilon p^{*}+K_{3}}\right), \quad G^{*}=\Upsilon \exp \left(-\Upsilon x^{*}\right)
$$

Henceforth, we drop the star superscript from the dimensionless variables.

\subsection{Parameter estimates for real devices}

A list of parameter estimates obtained from the literature is supplied in Table 1. Note that $F_{p h}, \tau_{n}, \tau_{p}$ and $D_{+}$are in line with the range of values found in the literature but have been specifically chosen to give good agreement to the experimental $J-V$ curves presented by Richardson et al. [28]. Based on this data, the dimensionless parameters, corresponding 
to a cell with perovskite width $b=600 \mathrm{~nm}$, are

$$
\begin{array}{lll}
\lambda=2.4 \times 10^{-3}, & v=5.8 \times 10^{-10}, & \delta=2.1 \times 10^{-7}, \\
\kappa_{n}=\kappa_{p}=1, & \Delta=0, & \epsilon=3.3 \times 10^{-3}, \\
\bar{p}=0.30, & \bar{n}=20, & N_{i}=8.6 \times 10^{-9}, \\
\gamma=2.4, & K_{3}=8.6 \times 10^{-9}, & \Upsilon=3.7 .
\end{array}
$$

While, for a cell with perovskite width $b=150 \mathrm{~nm}$, they remain unchanged except that

$$
\begin{aligned}
& \lambda=1.0 \times 10^{-2}, \quad \quad v=1.4 \times 10^{-10}, \quad \delta=5.2 \times 10^{-8}, \\
& \bar{p}=1.2, \quad \bar{n}=82, \quad N_{i}=3.4 \times 10^{-8}, \\
& \gamma=0.15, \quad K_{3}=3.5 \times 10^{-8}, \quad Y=0.92 \text {. }
\end{aligned}
$$

For the range of possible perovskite layer thicknesses considered, it always holds that $\delta \ll \lambda \ll 1$ and this observation motivates the asymptotic solution to the model considered in the next section.

\section{Asymptotic simplification of the model $(\delta \ll \lambda \ll 1)$}

Here, we assume dimensionless parameter sizes consistent with (2.16) and in particular require that $\delta \ll \lambda \ll 1$. In this scenario, the problem for the anion vacancy density and potential $(P$ and $\phi)$ decouples from that for the charge carrier densities ( $n$ and $p$ ) so that a very good estimate of $\phi$ can be obtained by ignoring the contributions of $n$ and $p$ in the last equation of (2.12). We shall further assume that the cation vacancies are effectively immobile on the timescales of interest, reflected in the choice of $\Delta=D_{-} / D_{+}=0$. This assumption, coupled to equations (2.12) and initial conditions (2.14), imply that the cation vacancy density remains constant with $N \equiv 1$.

\subsection{The ion problem}

A good approximation to the potential can be obtained from the ion vacancy dependent equations in (2.12)-(2.14) at leading order, i.e.,

$$
\begin{aligned}
\frac{\partial P}{\partial t}+\lambda \frac{\partial \mathcal{F}_{p}}{\partial x} & =0, \quad \mathcal{F}_{p}=-\left(\frac{\partial P}{\partial x}+P \frac{\partial \phi}{\partial x}\right), \\
\frac{\partial^{2} \phi}{\partial x^{2}} & =\frac{1}{\lambda^{2}}(1-P)
\end{aligned}
$$

with

$$
\begin{aligned}
\left.\phi\right|_{x=0}=\frac{\Phi_{b i}-\Phi}{2}, & \left.\mathcal{F}_{p}\right|_{x=0}=0, \\
\left.\phi\right|_{x=1}=-\frac{\Phi_{b i}-\Phi}{2}, & \left.\mathcal{F}_{p}\right|_{x=1}=0,\left.\quad P\right|_{t=0}=1 .
\end{aligned}
$$

Since $\lambda \ll 1$, these equations can be further approximated by using asymptotic boundary layer theory, in a similar vein to Richardson et al. [27]. In the limit $\lambda \rightarrow 0$, the solution can 


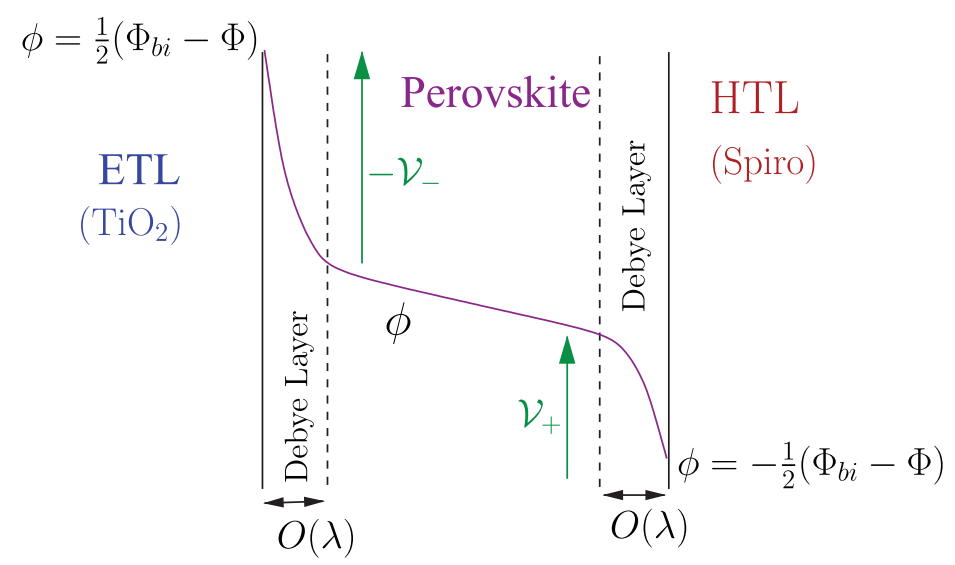

FIGURE 2. Schematic representation of the Debye layers and the solution for the electric potential, $\phi$.

be subdivided into three regions consisting of a bulk (or outer) region which is separated from the two boundaries by boundary layers of width $O(\lambda)$, see Figure 2. As is usual in this type of problem, these boundary layers are termed either Debye layers or double layers (we opt for the former usage).

\section{Bulk region}

Away from the boundaries (i.e., for $x \gg \lambda$ and $1-x \gg \lambda$ ), the variables $P, \mathcal{F}_{p}$ and $\phi$ can be expanded, in powers of $\lambda$ and $\delta$, as follows:

$$
P=1+\cdots, \quad \mathcal{F}_{p}=\mathcal{F}_{p, 0}^{(o)}+\cdots, \quad \phi=\phi_{0}^{(o)}+\cdots .
$$

Substituting these expansions into (3.1), and assuming $\delta / \lambda \ll 1$, gives, at leading order,

$$
\frac{\partial \mathcal{F}_{p, 0}^{(o)}}{\partial x}=0, \quad \mathcal{F}_{p, 0}^{(o)}=-\frac{\partial \phi_{0}^{(o)}}{\partial x} .
$$

Note that correction terms in the expansions of $P$ and $\phi$ are $O(\delta)$ and $O(\delta / \lambda)$, respectively. These arise from the presence of the $O(\delta)$ charge carrier terms in Poisson's equation (last of (2.12)) and this is why the expansion breaks down if the value of either $n$ or $p$ becomes comparable to $O(\lambda / \delta)$. It follows that $\phi_{0, x x}^{(o)}=0$ and hence that

$$
\phi_{0}^{(o)}=W_{-}(t)(1-x)+W_{+}(t) x
$$

for arbitrary functions of time $W_{-}(t)$ and $W_{+}(t)$. It follows, on substituting into (3.4), that the leading order ion flux is given by

$$
\mathcal{F}_{p, 0}^{(o)}=W_{-}(t)-W_{+}(t)
$$




\section{The Debye layers}

The asymptotic solution in the Debye layer about $x=0$ is obtained by rescaling space in the governing equations (3.1) and (3.2) via

$$
x=\lambda \zeta
$$

and substituting the asymptotic expansions

$$
P=P_{0}^{(d)}(\zeta, t)+\cdots, \quad \mathcal{F}_{p}=\mathcal{F}_{p, 0}^{(d)}(\zeta, t)+\cdots, \quad \phi=\phi_{0}^{(d)}(\zeta, t)+\cdots,
$$

into the rescaled equations to obtain the leading order problem. The solution to which is given in the appendix and can be summarised as follows: (A) the leading order potential, $\phi_{0}^{(d)}(\zeta, t)$, and vacancy distribution, $P_{0}^{(d)}(\zeta, t)$, are both quasi-steady throughout the Debye layer, (B) the vacancy distribution is in quasi-equilibrium and so is Boltzmann distributed and $(\mathrm{C})$ the potential satisfies a modified version of the Poisson-Boltzmann equation. The solution to this problem can be written in the form

$$
\begin{aligned}
& P_{0}^{(d)}(\zeta, t)=\exp \left(-\theta\left(\zeta, \mathcal{V}_{-}(t)\right)\right) \\
& \phi_{0}^{(d)}(\zeta, t)=\theta\left(\zeta, \mathcal{V}_{-}(t)\right)+W_{-}(t)
\end{aligned}
$$

where $W_{-}$is the potential at the left-hand side of the bulk, to which $\phi_{0}^{(d)}$ matches as $\zeta \rightarrow+\infty$, and $\mathcal{V}_{-}(t)$ is the potential drop across the Debye layer (see Figure 2). The function $\theta\left(\zeta, \mathcal{V}_{-}\right)$is defined by the solution $\theta(z, \mathcal{V})$ to the generic modified PoissonBoltzmann problem

$$
\frac{\partial^{2} \theta}{\partial z^{2}}=1-e^{-\theta},\left.\quad \theta\right|_{z=0}=-\mathcal{V}, \quad \theta \rightarrow 0, z \rightarrow \infty .
$$

Similarly, the asymptotic solution in the Debye layer about $x=1$ is obtained by rescaling space in the governing equations (3.1) and (3.2) using the transformation

$$
x=1-\lambda \xi
$$

and substituting the asymptotic expansions

$$
P=P_{0}^{(D)}(\xi, t)+\cdots, \quad \mathcal{F}_{p}=\mathcal{F}_{p, 0}^{(D)}(\xi, t)+\cdots, \quad \phi=\phi_{0}^{(D)}(\xi, t)+\cdots,
$$

into the resulting equations and solving at leading order. Once again this process is described in detail in the appendix. As in the other Debye layer, the leading order potential $\phi_{0}^{(D)}(\xi, t)$ and vacancy distribution $P_{0}^{(D)}(\xi, t)$ can be written in the form

$$
\begin{aligned}
& P_{0}^{(D)}(\xi, t)=\exp \left(-\theta\left(\xi, \mathcal{V}_{+}(t)\right)\right), \\
& \phi_{0}^{(D)}(\xi, t)=\theta\left(\xi, \mathcal{V}_{+}(t)\right)+W_{+}(t),
\end{aligned}
$$

where $W_{+}$is the potential at the right-hand side of the bulk (to which $\phi_{0}^{(D)}$ matches as $\xi \rightarrow+\infty), \mathcal{V}_{+}(t)$ is the potential drop across this Debye layer (see Figure 2) and $\theta\left(\xi, \mathcal{V}_{+}(t)\right.$ ) is once again a solution to the problem (3.9). 
In order to fully determine the leading order solutions in both Debye layers and the bulk region, it is necessary to solve for the time-dependent functions $\mathcal{V}_{-}, W_{-}, \mathcal{V}_{+}$and $W_{+}$. The requirement that the leading order solutions in the Debye layers, (3.8) and (3.12), satisfy the potential boundary conditions in (3.2) gives

$$
W_{-}(t)-\mathcal{V}_{-}(t)=\frac{\Phi_{b i}-\Phi(t)}{2}, \quad W_{+}(t)-\mathcal{V}_{+}(t)=-\frac{\Phi_{b i}-\Phi(t)}{2}
$$

\section{Charge conservation within the Debye layers}

A further two conditions on these four functions can be obtained by matching the flux of vacancies into the Debye layers with the leading order expansion of the vacancy conservation equations in the Debye layers. This leads to solvability conditions (described in appendix) which can be interpreted in terms of global conservation of charge within the Debye layers. Since the leading order solutions for the vacancy densities within the Debye layers are quasi-steady, the total (dimensionless) charge per unit area within each Debye layer, $\mathcal{Q}$, can be related to the potential drop across the layer, $\mathcal{V}$, in the form of a non-linear capacitance relation. Here, the charges per unit area contained within each Debye layer $\left(\mathcal{Q}_{-}\right.$in that about $x=0$ and $\mathcal{Q}_{+}$in that about $\left.x=1\right)$ are defined, in terms of the local Debye layer variables $\zeta$ and $\xi$, by

$$
\mathcal{Q}_{-}=\int_{0}^{\infty}(P-1) \mathrm{d} \zeta, \quad \mathcal{Q}_{+}=\int_{0}^{\infty}(P-1) \mathrm{d} \xi,
$$

and, as shown in the appendix, are related to the potential drops across the Debye layers $\left(\mathcal{V}_{-}\right.$and $\mathcal{V}_{+}$, respectively) via the capacitance relations

$$
\mathcal{Q}_{-}=Q\left(\mathcal{V}_{-}(t)\right), \quad \mathcal{Q}_{+}=Q\left(\mathcal{V}_{+}(t)\right)
$$

where the function $Q(\mathcal{V})$ is defined by

$$
Q(\mathcal{V})=\operatorname{sign}(\mathcal{V})\left(2\left(e^{\mathcal{V}}-1-\mathcal{V}\right)\right)^{1 / 2}
$$

This relation is plotted in Figure 3.

Furthermore, since vacancies (and hence charge) are conserved, the rate of change of the total charge per unit area within the Debye layers must equal the flux of (positively charged) vacancies flowing into each layer from the bulk region. Since the vacancy flux in the bulk region, $\mathcal{F}_{p, 0}^{(o)}$, is spatially independent, and given by (3.5), this observation corresponds to the conditions

$$
\frac{d \mathcal{Q}_{-}}{d t}=W_{+}(t)-W_{-}(t), \quad \frac{d \mathcal{Q}_{+}}{d t}=W_{-}(t)-W_{+}(t) .
$$

Alternatively, on eliminating $W_{+}$and $W_{-}$in favour of $\mathcal{V}_{+}$and $\mathcal{V}_{-}$, we have the equivalent conditions

$$
\begin{aligned}
& \frac{d \mathcal{Q}_{-}}{d t}=-\left[\Phi_{b i}-\Phi(t)+\mathcal{V}_{-}(t)-\mathcal{V}_{+}(t)\right] \\
& \frac{d \mathcal{Q}_{+}}{d t}=\Phi_{b i}-\Phi(t)+\mathcal{V}_{-}(t)-\mathcal{V}_{+}(t)
\end{aligned}
$$




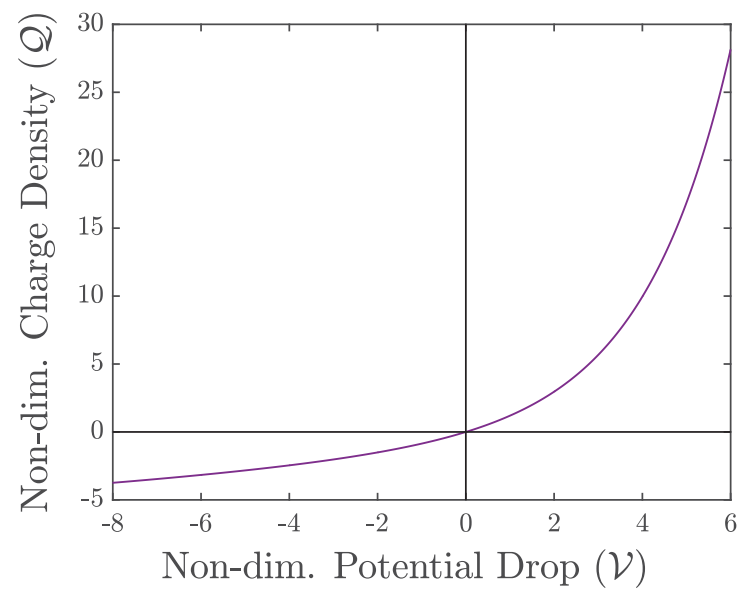

FIGURE 3. Charge density in the Debye layer, $\mathcal{Q}$, versus the potential drop across it, $\mathcal{V}$, defined by (3.16), or equivalently (3.19).

which can be solved in conjunction with (3.15) and (3.16). Adding these two equations together and integrating with respect to $t$ implies that the total charge in the Debye layers is conserved, i.e., $\mathcal{Q}_{-}(t)+\mathcal{Q}_{+}(t)$ is constant. This is to be expected given that the predominant mobile charge carriers are the positive vacancies which cannot leave the perovskite region. Furthermore, since the net charge arising from both positive and negative vacancies will initially always be zero, it remains so for all time, i.e.,

$$
\mathcal{Q}_{-}(t)=-\mathcal{Q}_{+}(t)
$$

At this stage, we can choose either to solve an ODE for $\mathcal{V}_{+}(t)$ or one for $\mathcal{Q}_{+}(t)$. Since neither of these problems admit exact solution, we opt to solve for $\mathcal{Q}_{+}$because this is preferable from a numerical point of view. We do this by noting that the inverse of (3.16) is

$$
\mathcal{V}(Q)=\left\{\begin{array}{c}
-\log _{e}\left(-\frac{1}{\operatorname{LambertW}_{0}\left(-\exp \left(-\left(Q^{2} / 2+1\right)\right)\right)}\right) \\
\text { for } Q<0, \\
\log _{e}\left(-\mathrm{LambertW}_{-1}\left(-\exp \left(-\left(Q^{2} / 2+1\right)\right)\right)\right) \\
\text { for } Q>0,
\end{array}\right.
$$

where $\operatorname{LambertW}_{0}(\cdot)$ and $\mathrm{LambertW}_{-1}(\cdot)$ are the 0 'th and -1 'st branch of the Lambert $\mathrm{W}$ function. On substituting the above functional relation in (3.17), together with (3.18), we obtain a single ODE for $\mathcal{Q}_{+}(t)$

$$
\frac{\mathrm{d} \mathcal{Q}_{+}}{\mathrm{d} t}=\Phi_{b i}-\Phi(t)+\mathcal{V}\left(-\mathcal{Q}_{+}\right)-\mathcal{V}\left(\mathcal{Q}_{+}\right)
$$

The solution to (3.20) may be used to obtain the leading order bulk potential via (3.4), that is,

$$
\begin{array}{r}
\phi_{0}^{(o)}(x, t)=(1-x)\left(\mathcal{V}\left(-\mathcal{Q}_{+}(t)\right)+\frac{1}{2}\left[\Phi_{b i}-\Phi(t)\right]\right) \\
+x\left(\mathcal{V}\left(\mathcal{Q}_{+}(t)\right)-\frac{1}{2}\left[\Phi_{b i}-\Phi(t)\right]\right)
\end{array}
$$




\section{Remark}

The dimensional surface charge density (in the Debye layers), $\mathcal{Q}^{\text {(dim) }}$, is related to its non-dimensional counterpart, $\mathcal{Q}$, by

$$
\mathcal{Q}^{(\operatorname{dim})}=q \lambda b N_{0} \mathcal{Q} .
$$

\section{The uniformly valid approximation to $\phi$}

We can now write down a uniformly valid approximation to $\phi$ that is valid throughout the bulk and both Debye layers:

$$
\begin{aligned}
\phi \sim & (1-x)\left(\mathcal{V}\left(-\mathcal{Q}_{+}(t)\right)+\frac{1}{2}\left[\Phi_{b i}-\Phi(t)\right]\right)+x\left(\mathcal{V}\left(\mathcal{Q}_{+}(t)\right)-\frac{1}{2}\left[\Phi_{b i}-\Phi(t)\right]\right) \\
& +\theta\left(\frac{x}{\lambda}, \mathcal{V}\left(-\mathcal{Q}_{+}(t)\right)\right)+\theta\left(\frac{1-x}{\lambda}, \mathcal{V}\left(\mathcal{Q}_{+}(t)\right)\right),
\end{aligned}
$$

where the function $\theta(z, \mathcal{V})$ is defined implicitly in (15) in the appendix. The corresponding uniformly valid asymptotic approximation for the anion vacancy density, $P$, is

$$
P \sim \exp \left(-\theta\left(\frac{x}{\lambda}, \mathcal{V}\left(-\mathcal{Q}_{+}(t)\right)\right)\right)+\exp \left(-\theta\left(\frac{1-x}{\lambda}, \mathcal{V}\left(\mathcal{Q}_{+}(t)\right)\right)\right)-1
$$

\subsection{Asymptotic approximation to the charge carrier equations}

As we demonstrate in Section 4, the potential is well-approximated by the solution to the ion problem (3.1) and (3.2) and is almost entirely unaffected by the carrier distributions. Furthermore, since the Debye layers are extremely thin, the effects of both photo-generation and recombination within these layers are negligible so that, from (2.12), the electron and hole currents are to a good approximation spatially independent across these layers,

$$
j_{p}^{(d)} \approx j_{p}^{(d)}(t), \quad j_{n}^{(d)} \approx j_{n}^{(d)}(t), \quad j_{p}^{(D)} \approx j_{p}^{(D)}(t), \quad j_{n}^{(D)} \approx j_{n}^{(D)}(t) .
$$

Furthermore, in these narrow regions, electron and hole densities are in approximate quasi-thermal equilibrium. In particular, in the Debye layers close to $x=0$ and $x=1$, respectively,

$$
\frac{\partial n^{(d)}}{\partial \zeta} \sim n^{(d)} \frac{\partial \phi_{0}^{(d)}}{\partial \zeta}, \quad \frac{\partial p^{(D)}}{\partial \xi} \sim-p^{(D)} \frac{\partial \phi_{0}^{(D)}}{\partial \xi}
$$

Referring to the boundary conditions (2.13), we find that

$$
\begin{aligned}
& n^{(d)} \sim \bar{n} \exp \left(\phi_{0}^{(d)}-\frac{1}{2}\left(\Phi_{b i}-\Phi\right)\right) \text { near } x=0, \\
& p^{(D)} \sim \bar{p} \exp \left(-\phi_{0}^{(D)}-\frac{1}{2}\left(\Phi_{b i}-\Phi\right)\right) \text { near } x=1 .
\end{aligned}
$$


For the purposes of predicting the output current of the device, we need only determine the carrier concentrations within the bulk region. Matching conditions on the bulk carrier problems (for $n$ and $p$ ) are obtained from the far-field behaviour of the Debye layer solutions, namely,

$$
\begin{aligned}
& n^{(d)} \rightarrow \bar{n} \exp \left(\mathcal{V}_{-}(t)\right) \text { as } \zeta \rightarrow+\infty, \\
& p^{(D)} \rightarrow \bar{p} \exp \left(-\mathcal{V}_{+}(t)\right) \text { as } \xi \rightarrow+\infty
\end{aligned}
$$

The appropriate boundary conditions on the bulk carrier densities are thus

$$
\left.\left.\begin{array}{l}
n^{(o)}=\bar{n} \exp \left(\mathcal{V}_{-}(t)\right) \\
j_{p}^{(o)}=-R_{l}
\end{array}\right\} x=0^{+}, \quad \begin{array}{l}
p^{(o)}=\bar{p} \exp \left(-\mathcal{V}_{+}(t)\right) \\
j_{n}^{(o)}=-R_{r}
\end{array}\right\} x=1^{-} .
$$

The corresponding equations for the carrier densities in the bulk, as obtained from (2.12), are, on taking the physically appropriate limit $v \rightarrow 0$,

$$
\begin{array}{lc}
\frac{\partial j_{p}^{(o)}}{\partial x}=G-R\left(n^{(o)}, p^{(o)}\right), & j_{p}^{(o)}=-\kappa_{p}\left(\frac{\partial p^{(o)}}{\partial x}-p^{(o)} E_{0}^{(o)}\right), \\
\frac{\partial j_{n}^{(o)}}{\partial x}=-G+R\left(n^{(o)}, p^{(o)}\right), & j_{n}^{(o)}=\kappa_{n}\left(\frac{\partial n^{(o)}}{\partial x}+n^{(o)} E_{0}^{(o)}\right),
\end{array}
$$

where $E_{0}^{(o)}(t)$ is the leading order bulk electric field defined by $E_{0}^{(o)}(t)=-\partial \phi_{0}^{(o)} / \partial x$ and, from (3.21), is given by

$$
E_{0}^{(o)}(t)=\mathcal{V}_{-}(t)-\mathcal{V}_{+}(t)+\Phi_{b i}-\Phi(t)
$$

Hence, the asymptotic approximation to the charge carrier problem can be found from the solution of (3.24) and (3.25) in which the electric field term, $E_{0}^{(o)}(t)$, depends, via (3.26), on the solution $\mathcal{Q}_{+}(t)$ to the ion problem, through the relations $\mathcal{V}_{-}=\mathcal{V}\left(-\mathcal{Q}_{+}\right)$ and $\mathcal{V}_{+}=\mathcal{V}\left(\mathcal{Q}_{+}\right)$(where the function $\mathcal{V}(\cdot)$ is defined in (3.19)). Usually, the solution will have to be obtained numerically because of the non-linearity of the recombination term. Nonetheless, numerically solving this reduced problem is considerably less challenging than directly tackling (2.12)-(2.14) because it excludes the Debye layers, over which the solution varies very rapidly. Finally, we note that the net current density $j^{(o)}(t)=j_{n}^{(o)}(x, t)+j_{p}^{(o)}(x, t)$ is independent of the spatial variable $x$ and so can be found simply by evaluating the sum of the electron and hole current densities at any point in the domain.

\subsection{An analytic solution in the limit $\epsilon \rightarrow 0$ with zero interfacial recombination}

It is notable that the parameter $\epsilon=\tau_{n} / \tau_{p}$ is typically small (we estimate, on the basis of earlier work [28], $\epsilon \approx 3.3 \times 10^{-3}$ ), while the other parameters in the SRH recombination term (2.15), $N_{i}$ and $K_{3}$, are both very small. These observations lead us to set $N_{i} \equiv 0$, $K_{3} \equiv 0$ and to investigate the small $\epsilon$ limit. In which case, provided that $p / n$ is not large, $R(n, p)$ can be approximated by

$$
R(n, p) \sim \gamma p
$$


If we restrict our interest to the case where interfacial recombination is negligible (i.e., if we take $R_{l} \equiv 0$ and $R_{r} \equiv 0$ ), it follows that the equation for the hole density decouples from that for the electron density (see (3.25)) and can be reformulated as the following linear equation for $p^{(o)}$ :

$$
\frac{\partial j_{p}^{(o)}}{\partial x}=\Upsilon \exp (-\Upsilon x)-\gamma p^{(o)}, \quad \frac{\partial p^{(o)}}{\partial x}-p^{(o)} E_{0}^{(o)}=-\frac{j_{p}^{(o)}}{\kappa_{p}} .
$$

These may be solved by eliminating $j_{p}^{(o)}$ from the above to obtain a second-order constant coefficient linear inhomogeneous equation for $p_{0}^{(o)}$, namely,

$$
\frac{\partial^{2} p^{(o)}}{\partial x^{2}}-E_{0}^{(o)} \frac{\partial p^{(o)}}{\partial x}-\frac{\gamma p^{(o)}}{\kappa_{p}}=-r \exp (-\Upsilon x)
$$

This can be rewritten in the form

$$
\frac{\partial^{2} p^{(o)}}{\partial x^{2}}-\left(\beta_{1}(t)+\beta_{2}(t)\right) \frac{\partial p^{(o)}}{\partial x}+\beta_{1}(t) \beta_{2}(t) p^{(o)}=-d \exp (-\Upsilon x),
$$

where

$$
\begin{aligned}
\beta_{1}(t) & =\frac{E_{0}^{(o)}(t)}{2}+\frac{\left(\left(E_{0}^{(o)}(t)\right)^{2}+4 \gamma / \kappa_{p}\right)^{1 / 2}}{2}, \\
\beta_{2}(t) & =\frac{E_{0}^{(o)}(t)}{2}-\frac{\left(\left(E_{0}^{(o)}(t)\right)^{2}+4 \gamma / \kappa_{p}\right)^{1 / 2}}{2}, \\
d & =\frac{\Upsilon}{\kappa_{p}} .
\end{aligned}
$$

On noting that $E_{0}^{(o)}(t)=\beta_{1}(t)+\beta_{2}(t)$, the boundary conditions (3.24) can be stated as

$$
\begin{array}{r}
\frac{\partial p^{(o)}}{\partial x}-\left.p^{(o)}\left(\beta_{1}(t)+\beta_{2}(t)\right)\right|_{x=0}=0 \\
\left.p^{(o)}\right|_{x=1}=\bar{p} \exp \left(-\mathcal{V}_{+}(t)\right)
\end{array}
$$

The solution to (3.28) and (3.30) is

$$
p^{(o)}(x, t)=-\frac{d e^{-\Upsilon x}}{\left(\Upsilon+\beta_{1}(t)\right)\left(\Upsilon+\beta_{2}(t)\right)}+\mathcal{A}(t) e^{\beta_{1}(t) x}+\mathcal{B}(t) e^{\beta_{2}(t) x},
$$

where

$$
\begin{aligned}
& \mathcal{A}(t)=\frac{\hat{\mathcal{A}}(t)}{\mathcal{D}(t)}, \quad \mathcal{B}(t)=\frac{\hat{\mathcal{B}}(t)}{\mathcal{D}(t)}, \quad \mathcal{D}(t)=\beta_{1}(t) e^{\beta_{1}(t)}-\beta_{2}(t) e^{\beta_{2}(t)}, \\
& \hat{\mathcal{A}}(t)=\beta_{1}(t) \bar{p} \exp \left(-\mathcal{V}_{+}(t)\right)-\frac{d\left(e^{\beta_{2}(t)}\left(\beta_{1}(t)+\beta_{2}(t)+\Upsilon\right)-\beta_{1}(t) e^{-\Upsilon}\right)}{\left(\Upsilon+\beta_{1}(t)\right)\left(\Upsilon+\beta_{2}(t)\right)}, \\
& \hat{\mathcal{B}}(t)=-\beta_{2}(t) \bar{p} \exp \left(-\mathcal{V}_{+}(t)\right)-\frac{d\left(e^{\beta_{1}(t)}\left(\beta_{1}(t)+\beta_{2}(t)+\Upsilon\right)-\beta_{2}(t) e^{-\Upsilon}\right)}{\left(\Upsilon+\beta_{1}(t)\right)\left(\Upsilon+\beta_{2}(t)\right)} .
\end{aligned}
$$


An expression for the total current in the device

An expression for the hole current density $j_{p}^{(o)}$ is found by substituting the solution (3.31) for $p^{(o)}$ into (3.25); this gives

$$
j_{p}^{(o)}=-\kappa_{p}\left(d e^{-\Upsilon x} \frac{\left(\Upsilon+\beta_{1}(t)+\beta_{2}(t)\right)}{\left(\Upsilon+\beta_{1}(t)\right)\left(\Upsilon+\beta_{2}(t)\right)}-\beta_{2}(t) \mathcal{A}(t) e^{\beta_{1}(t) x}-\beta_{1}(t) \mathcal{B}(t) e^{\beta_{2}(t) x}\right) .
$$

The total current $J(t)=j_{p}^{(o)}(x, t)+j_{n}^{(o)}(x, t)$ is determined from the condition that $j_{n}^{(o)}(1, t)=$ 0 , which thus implies that $J(t)=j_{p}^{(o)}(1, t)$. It follows that

$$
J(t)=-\kappa_{p}\left(d e^{-\Upsilon} \frac{\left(\Upsilon+\beta_{1}(t)+\beta_{2}(t)\right)}{\left(\Upsilon+\beta_{1}(t)\right)\left(\Upsilon+\beta_{2}(t)\right)}-\beta_{2}(t) \mathcal{A}(t) e^{\beta_{1}(t)}-\beta_{1}(t) \mathcal{B}(t) e^{\beta_{2}(t)}\right) .
$$

\section{Asymptotic solution for the bulk electron density}

In order to monitor whether this asymptotic solution breaks down, it is useful to derive an asymptotic expression for the bulk electron density, $n^{(o)}$, while recalling that we require $p^{(o)} / n^{(o)} \gg \epsilon$ in order for the validity of the expansion. The equations and boundary conditions for $n^{(o)}$ are, at leading order,

$$
\begin{aligned}
\frac{\partial n^{(o)}}{\partial x}+\left(\beta_{1}(t)+\beta_{2}(t)\right) n^{(o)} & =\frac{1}{\kappa_{n}}\left(J(t)-j_{p}^{(o)}\right), \\
\left.n^{(o)}\right|_{x=0} & =\bar{n} \exp \left(\mathcal{V}_{-}(t)\right),
\end{aligned}
$$

in which we once again write $E_{0}^{(o)}=\beta_{1}+\beta_{2}$ and where $j_{p}^{(o)}$ is given by (3.33). The solution to this problem is

$$
\begin{aligned}
n^{(o)}= & \bar{n} e^{\mathcal{V}_{-}(t)-\left(\beta_{1}(t)+\beta_{2}(t)\right) x}+\frac{J(t)}{\kappa_{n} E_{0}^{(o)}}\left(1-e^{-\left(\beta_{1}(t)+\beta_{2}(t)\right) x}\right) \\
& +\mathcal{D}(t)\left(e^{-Y x}-e^{-\left(\beta_{1}(t)+\beta_{2}(t)\right) x}\right)+\mathcal{G}(t)\left(e^{\beta_{1}(t) x}-e^{-\left(\beta_{1}(t)+\beta_{2}(t)\right) x}\right) \\
& +\mathcal{H}(t)\left(e^{\beta_{2}(t) x}-e^{-\left(\beta_{1}(t)+\beta_{2}(t)\right) x}\right),
\end{aligned}
$$

where time-dependent functions $\mathcal{D}, \mathcal{G}$ and $\mathcal{H}$ are given by

$$
\begin{aligned}
\mathcal{D}(t) & =\frac{\kappa_{p}}{\kappa_{n}}\left(\frac{d\left(\Upsilon+\beta_{1}(t)+\beta_{2}(t)\right)}{\left(\beta_{1}(t)+\beta_{2}(t)-\Upsilon\right)\left(\Upsilon+\beta_{1}(t)\right)\left(\Upsilon+\beta_{2}(t)\right)}\right), \\
\mathcal{G}(t) & =-\frac{\kappa_{p} \beta_{2}(t) \mathcal{A}(t)}{\kappa_{n}\left(2 \beta_{1}(t)+\beta_{2}(t)\right)}, \\
\mathcal{H}(t) & =-\frac{\kappa_{p} \beta_{1}(t) \mathcal{B}(t)}{\kappa_{n}\left(\beta_{1}(t)+2 \beta_{2}(t)\right)} .
\end{aligned}
$$

\section{Comparison between numerical and asymptotic solutions to the model}

In this section, we compare the results obtained from (i) a numerical solution to the full model, (2.12)-(2.14), to those obtained from (ii) a combined asymptotic/numerical approach, in which the ion problem is solved asymptotically as in Section 3.1, and from (iii) 
the special case described in Section 3.3, which is entirely based on asymptotic approximations. In particular, we show that the results from (ii) the combined asymptotic/numerical approach, adopted in an earlier work [28], compare extremely favourably to (i) numerical solution of the model.

\subsection{Numerical methods}

In approach (i), we use the method of lines. A detailed description of the numerical scheme is given by Courtier et al. [7], here, we restrict ourselves to a brief outline. The spatial derivatives in equations (2.12) are treated using a finite difference approach that is second-order accurate in space, both on the internal and boundary points, and chosen in such a way that conservation of species is also exact up to second order. After application of the finite difference approximations, the problem is reduced to a system of differential algebraic equations (DAEs) in which the ODEs arise from the evolution equations for $P, n$, and $p$, in (2.12), and the algebraic equations are a result of Poisson's equation for the potential. Solving systems of DAEs presents a challenging numerical problem, which we tackle using the ode15s routine in MATLAB [19]. Owing to rapid changes of the solution curves within the narrow Debye layers, we find that the problem is sufficiently stiff to require non-uniform grid spacing and the additional precision offered by Advanpix's Multiprecision Computing Toolbox [1].

In approach (ii), the system of equations requiring numerical treatment is that for the charge carriers in the bulk, (3.24) and (3.25). Having taken the asymptotic limits $\delta, \lambda$ and $v \rightarrow 0$, the remaining problem is a second-order boundary value problem (BVP). Crucially, since asymptotic expressions have been derived for the narrow Debye layers, only the solution in the bulk needs to be resolved numerically. This problem exhibits significantly reduced stiffness and, as a result, a straightforward application of the bvp4c routine in MATLAB [19] suffices.

\subsection{Results}

In Figures 4-7, we show results for a device characterised by the parameters given in Table 1 with the perovskite layer width equal to $600 \mathrm{~nm}$, corresponding to the set of dimensionless parameters given in (2.16). All numerical calculations are performed on a spatial grid consisting of 800 Chebyshev points.

Figures 4-6 show the internal state of a cell at five equally spaced values of time during a variation of the applied voltage, in a scenario in which the cell is abruptly illuminated at $t=0 \mathrm{~s}$ having been preconditioned in the dark with $V_{a p}=V_{b i}$. For Figure 4, the applied bias is varied smoothly from $V_{a p}=V_{b i}$ at $t=0 \mathrm{~s}$ to $V_{a p}=0.8 \mathrm{~V}$ at $t=10 \mathrm{~s}$ (precisely, $\left.V_{a p}=V_{b i}-0.2 \tanh (t) / \tanh (10)\right)$. Plots show solutions at $t=2,4,6,8,10 \mathrm{~s}$. For Figure 5, the applied bias is instantaneously decreased from $V_{a p}=V_{b i}$ to $V_{a p}=0 \mathrm{~V}$ at $t=0 \mathrm{~s}$ and held there for $4 \mathrm{~s}$. Plots show solutions at $t=0.8,1.6,2.4,3.2,4.0 \mathrm{~s}$. Finally, for Figure 6 , the applied bias is varied linearly from $V_{a p}=V_{b i}$ at $t=0 \mathrm{~s}$ to $V_{a p}=1.25 \mathrm{~V}$ at $t=10 \mathrm{~s}$. Plots are for $t=2,4,6,8,10 \mathrm{~s}$.

In Figure 7, comparison is made between current-voltage $(J-V)$ curves calculated using all three approaches and which model the experimental data presented by Richardson 

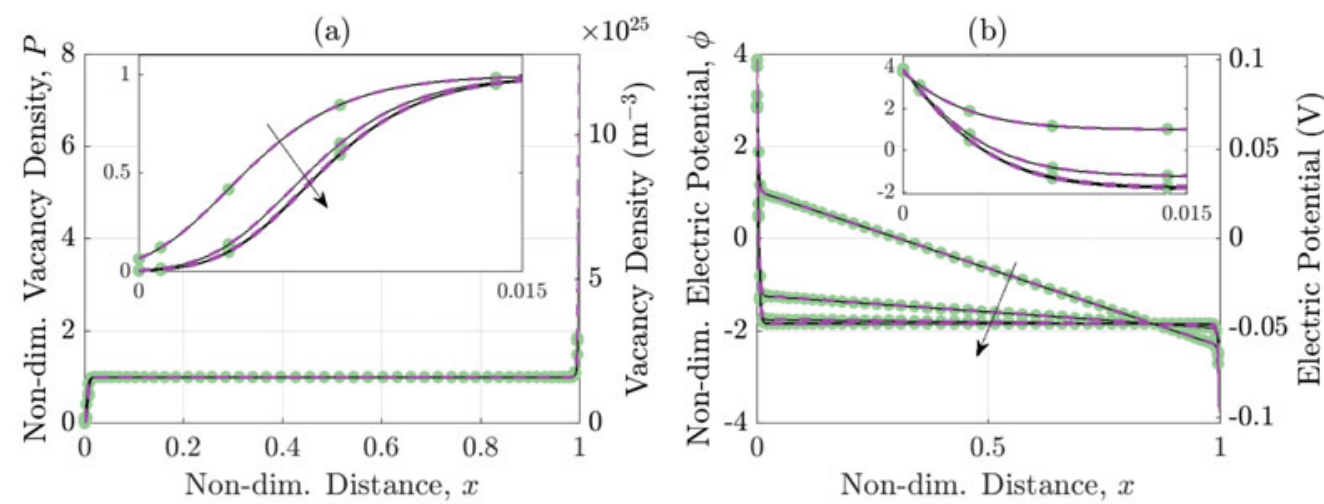

(c)

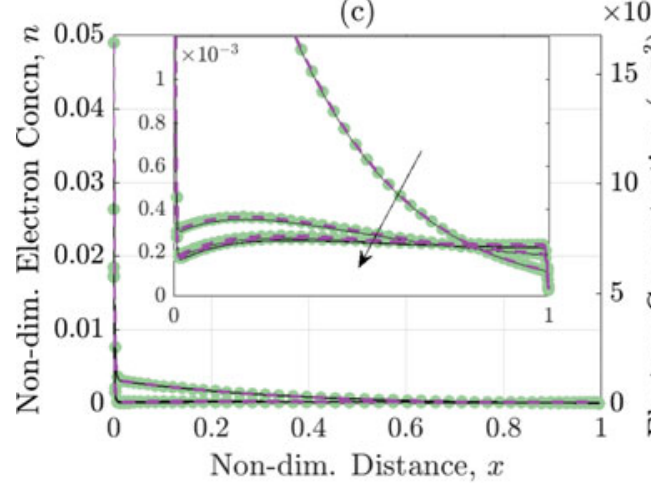

(d)

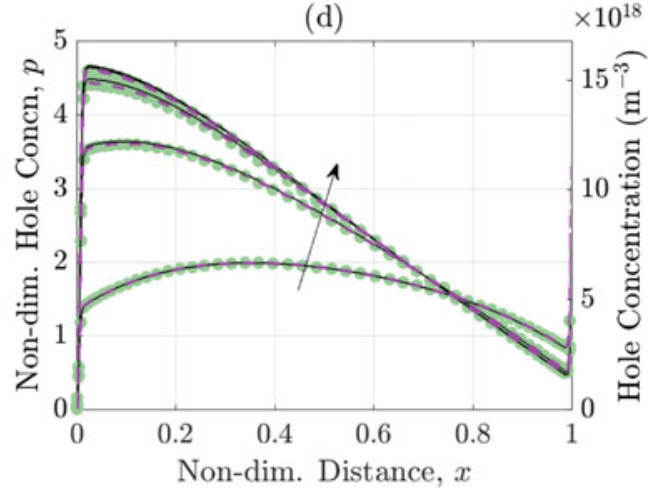

Figure 4. (a) Anion vacancy density, (b) electric potential, (c) electron concentration and (d) hole concentration profiles across the perovskite layer of a cell during a smooth decrease of applied bias from $V_{a p}=V_{b i}$ to $0.8 \mathrm{~V}$. Insets focus on the left-hand $\left(\mathrm{TiO}_{2} /\right.$ perovskite) boundary. Arrows indicate the direction of increasing time; black solid lines represent (i), the full numerical solutions, pink dashed lines represent (ii), the combined asymptotic/numerical approach and green circles represent (iii), the uniformly-valid asymptotic expansions from the fully asymptotic approach.

et al. [28]. In that paper, data is provided for two cells; here, we opt to model 'cell 2' and choose the colour scheme of Figure 7 for consistency with Figure 7(b) from [28]. The cell is preconditioned for $5 \mathrm{~s}$ at $1.2 \mathrm{~V}$ in the light before the $J-V$ curve is measured. The current is calculated at equally spaced intervals in time as the applied voltage is varied at a constant rate from $1.2 \mathrm{~V}$ (forward bias) to $0 \mathrm{~V}$ (short-circuit) and back; the four different scan rates are $50 \mathrm{mVs}^{-1}, 100 \mathrm{mVs}^{-1}, 250 \mathrm{mVs}^{-1}$ and $500 \mathrm{mVs}^{-1}$. In panel (a), solutions calculated using (i) the fully numerical (solid lines) and (ii) the combined asymptotic/numerical approach (dashed lines) are shown. Note that both of these methods calculate currents based on the full SRH recombination rate, (2.7). While in panel (b), solutions from (iii) the fully asymptotic approach are shown.

The agreement between the asymptotic and numerical results for electric potential and ion vacancy density presented in Figures 4-6 is extremely close in all cases. This suggests neglecting the effects of charge carriers (electrons and holes) on the electric potential is, in the physically pertinent regime considered here, a very good approximation. Agreement between the asymptotic and numerical results for the charge carrier densities ( $n$ and $p$ ) is 

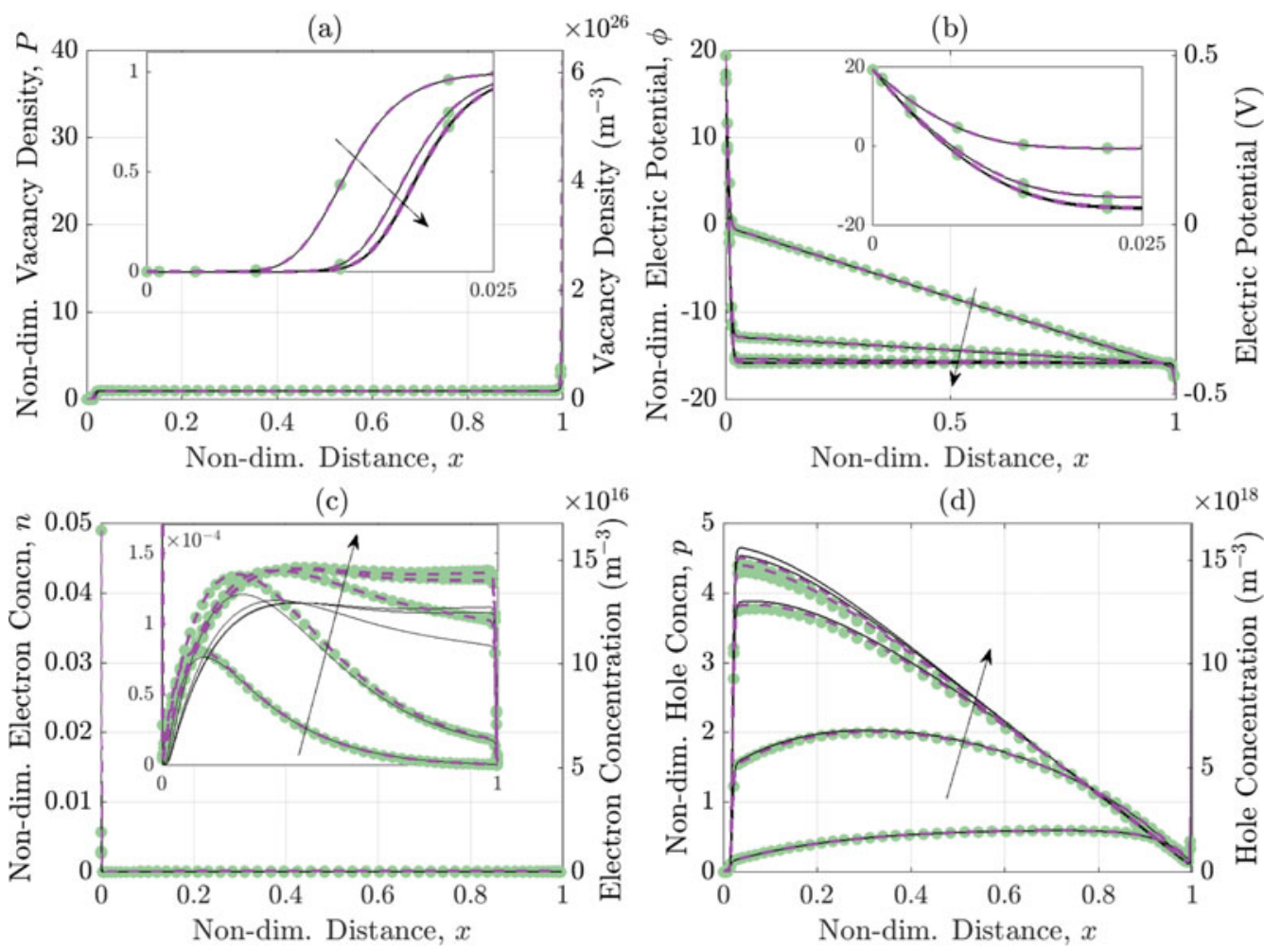

FIgURE 5. As for Figure 4 but for a fast evolution in applied bias from $V_{a p}=V_{b i}$ to $0 \mathrm{~V}$.

very good but exhibits some relatively minor discrepancies, particularly, in the case of the rapid transient (Figure 5).

The current-voltage curves produced in Figure 7, which follow typical experimental protocols, show minimal deviation between asymptotic and numerical results and go a long way to validating the approach we have adopted here as a useful tool in the study of these devices.

Estimates for the size of the typical ion vacancy density, $N_{0}$, in a PSC vary and, while our value is based on a reputable density functional calculation [10], we should allow for considerable variations in this quantity. In particular, it is interesting to ask the question of whether the asymptotic model we have derived still provides a good description of the physics in the case where $N_{0}$ is significantly smaller than our original estimate of $1.6 \times 10^{19} \mathrm{~cm}^{-3}$. In order to investigate this possibility, we consider the case where $N_{0}=1.6 \times 10^{17} \mathrm{~cm}^{-3}$ and compare solutions obtained using each of the three methods in the case of a voltage transient identical to the one investigated earlier in Figure 4. As can be seen in Figure 8, the agreement between the solution to the asymptotic model and the numerical solution of the full model is still extremely good despite $\lambda$ being 10 times larger than in the original calculations (here, $\lambda=2.4 \times 10^{-2}$ ). This indicates that the asymptotic approach taken in this work is robust with respect to significant variation in the size of the ion vacancy density $N_{0}$ and in particular is applicable to the range of ion vacancy densities commonly encountered in the literature. 

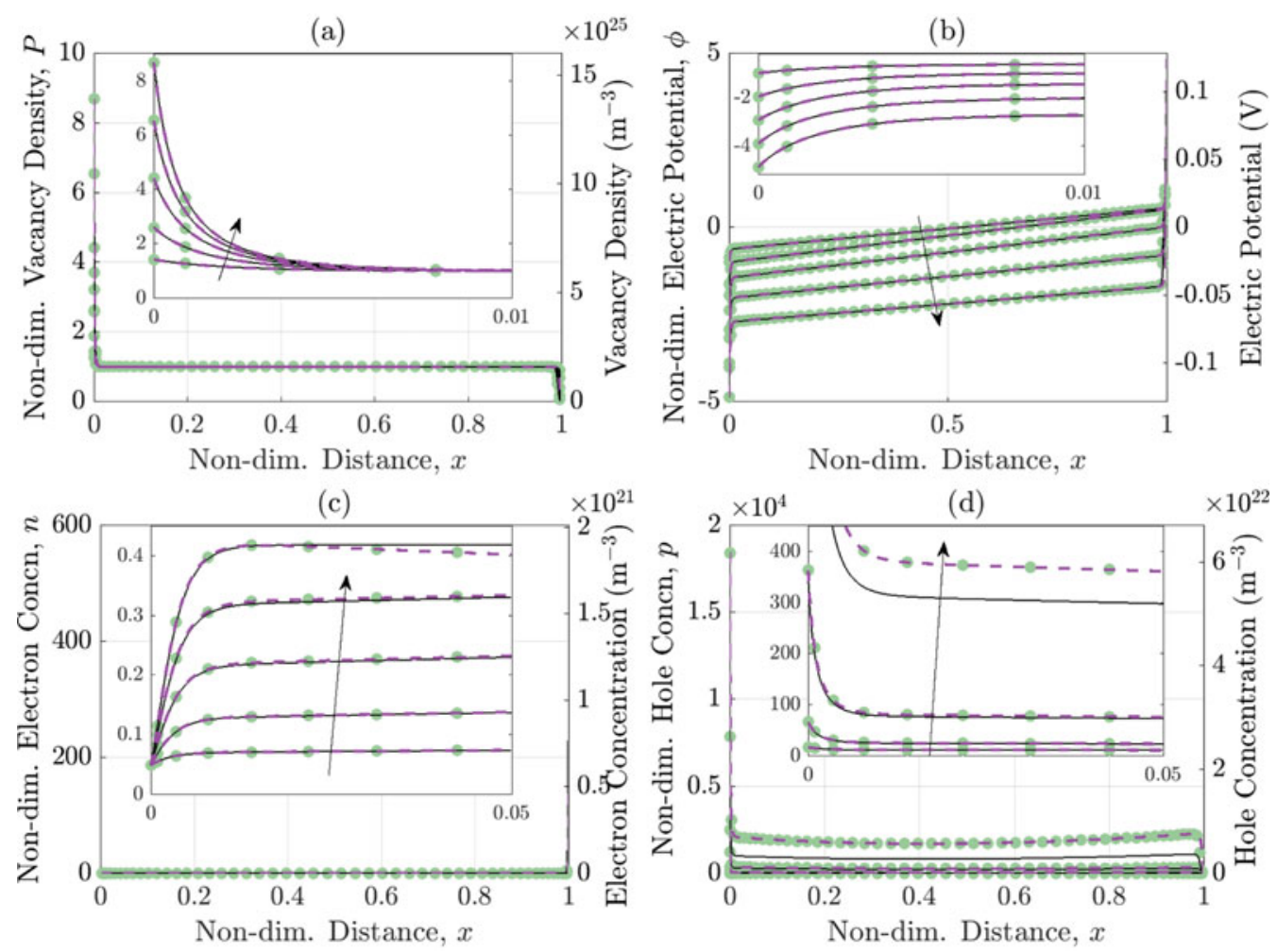

FIgURE 6. As for Figure 4 but for a slow increase in applied bias from $V_{a p}=V_{b i}$ to $1.2 \mathrm{~V}$.

\subsection{Discussion}

We have looked at three approaches to solving the drift-diffusion model (2.12)-(2.14). Approach (i) is fully numerical and involves solution of the full problem. In contrast, in approach (ii) (used previously [28]), we formally take the limits $\lambda \rightarrow 0$ (small Debye length) and $\delta \rightarrow 0$ (charge carrier concentration negligible in comparison to ion vacancy concentration). The comparison between the results of these two approaches is extremely favourable, as illustrated by the very small discrepancies in the $J-V$ curves calculated using both approaches, for a range of scan rates, in Figure 7(a).

The other main approximations to the drift-diffusion model that we make use of are the quasi-steady carrier limit, $v \rightarrow 0$ and the approximation of SRH recombination by hole dominated monomolecular recombination, $\epsilon \rightarrow 0$. The former limit $(v \rightarrow 0)$ and its use, or otherwise, makes negligible difference to the results obtained. The latter, however, is frequently problematic, despite the very small value of $\epsilon(=1 / 300)$ we use in the simulations. This slightly surprising result is best illustrated by the significant differences between $J-V$ curves calculated using the fully numerical method (solid curves in Figure 7(a)) and those calculated using the fully asymptotic method in the limit $\epsilon \rightarrow 0$ (Figure $7(b))$. Where there are significant differences between the two approaches this can be ascribed to strong spatial variations in charge carrier concentrations across the cell, 

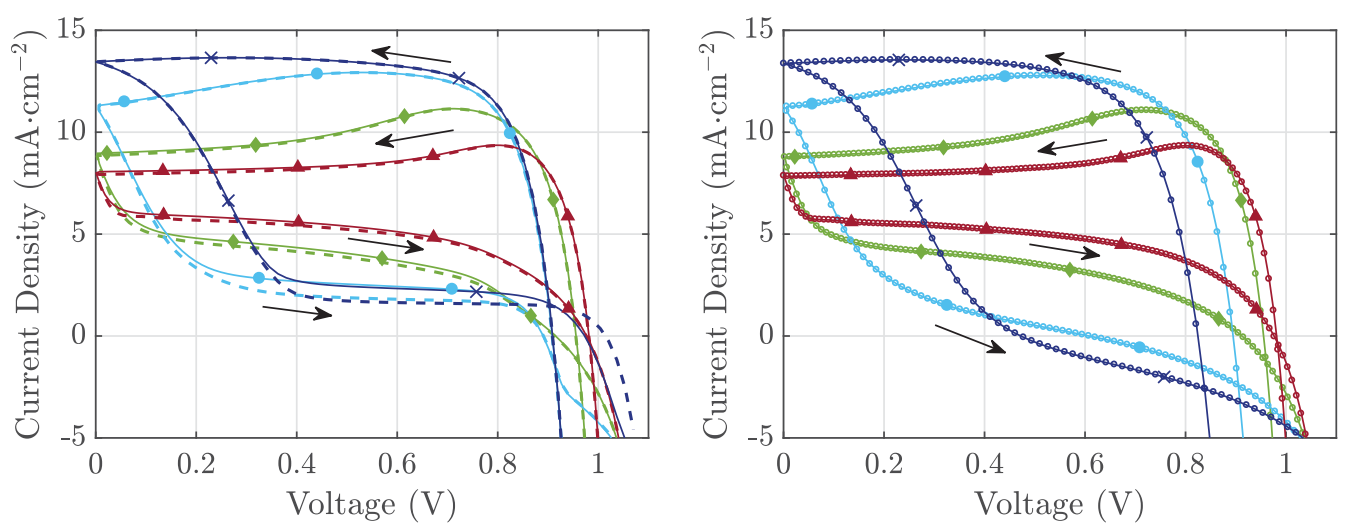

Figure 7. Comparison of solutions for $J-V$ curves at four different scan rates: $500 \mathrm{mVs}^{-1}$, blue with crosses; $250 \mathrm{mVs}^{-1}$, cyan with circles; $100 \mathrm{mVs}^{-1}$, green with diamonds; and $50 \mathrm{mVs}^{-1}$, red with triangles. Arrows show the direction of the voltage sweep.

resulting in regions where $n \leqslant O(\epsilon p)$ so that the approximation of $R(n, p)$ in (2.15) by $R(n, p) \approx \gamma p$ no longer holds.

\section{Conclusion}

In this work, we outlined a model for charge carrier transport and ion vacancy motion in a tri-layer planar PSC (previously discussed in Richardson et al. [28]). Using parameters extracted from the literature, we were able to identify two key small dimensionless parameters that characterise the model: $\lambda$, which gives the ratio of the Debye length in the perovskite to the width of perovskite layer, and $\delta$, the ratio of the typical charge carrier (electron and hole) densities to the typical ion vacancy density. Based on the small size of these parameters, we performed an asymptotic analysis of the model which showed that: (a) the problem for the ion vacancy density and the electric potential is almost completely independent of the charge carrier densities and (b) the decoupled problem for ion vacancies and electric potential is well-approximated by the solution to a single first-order ODE that describes the evolution of charge in the Debye layers (at the edge of the perovskite) in terms of the current through a resistor and a non-linear capacitor in series. In dimensional form, this simplified model states that the charge (per unit area) in the right-hand Debye layer, $\mathcal{Q}_{+}$, evolves according to the equation

$$
\frac{d \mathcal{Q}_{+}}{d t}=\frac{q D_{+} N_{0}}{V_{T}}\left(\frac{V_{b i}-V-\mathcal{V}\left(\mathcal{Q}_{+}\right)+\mathcal{V}\left(-\mathcal{Q}_{+}\right)}{b}\right),
$$

where the term in the brackets is the (uniform) electric field in the perovskite bulk (away from the Debye layers) and $\mathcal{V}(Q)$ is the inverse to the non-linear capacitance relation

$$
Q(\mathcal{V})=\frac{\varepsilon_{p} V_{T}}{L_{d}} \operatorname{sign}(\mathcal{V})\left[2\left(\exp \left(\mathcal{V} / V_{T}\right)-1-\frac{\mathcal{V}}{V_{T}}\right)\right]^{1 / 2}
$$





(c)

$\times 10^{16}$

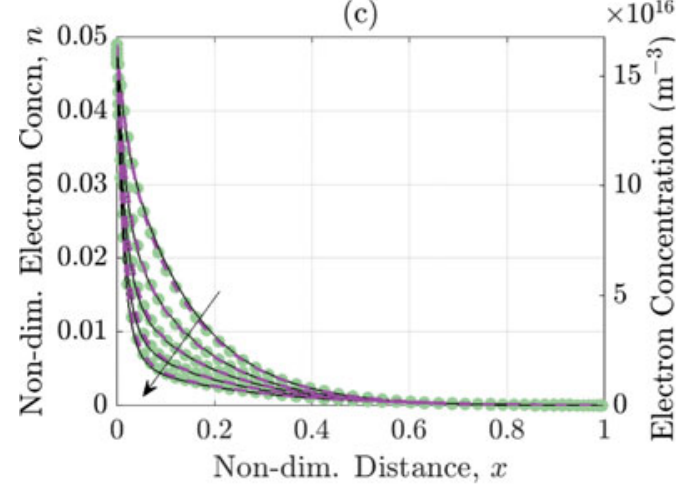

(d) $\times 10^{18}$

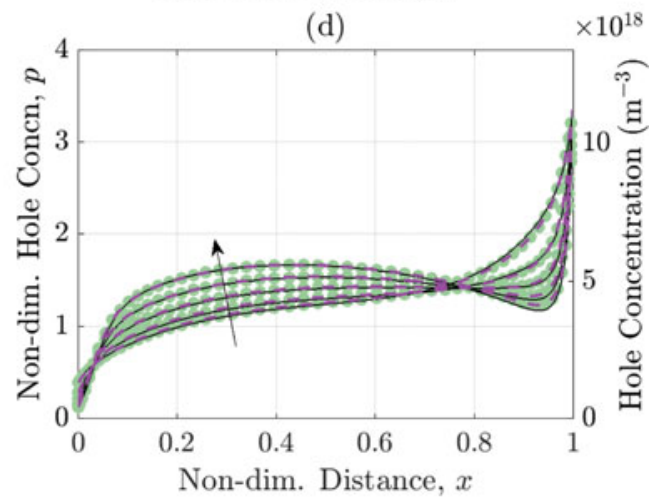

FIGURE 8. Equivalent to Figure 4 but for a cell with a lower vacancy density of $1.6 \times 10^{17} \mathrm{~cm}^{-3}$.

A good approximation to the full model can then be obtained by solving this much simplified problem for ion vacancy density and electric potential and using the resulting electric potential as an input into the charge carrier equations. The resulting model can sensibly be termed a surface polarisation model of charge transport because it describes the effect on the current in a cell of the polarisation of the perovskite layer, as ionic charge is transported from one of its surfaces to the other. In general, the simplified problem that we are left to solve for the charge carrier densities is non-linear and so requires numerical solution. However, in contrast to the problem for the ion vacancies and potential, it is non-stiff and so this is not usually problematic. Moreover, parameter estimates suggest that the Shockley-Read-Hall recombination term in the charge carrier equations can be well-approximated by monomolecular hole dominated recombination $(R(n, p) \approx \gamma p)$. This allows the charge carrier equations to be linearised and, in turn, solved analytically. Where this is the case, an asymptotic solution to the entire model can be obtained from the solution to the single first-order ODE discussed above.

In order to test the validity of the asymptotic method used to solve this model, we compared our asymptotic results to the results of a numerical solution of the full model. The latter was conducted using a recently developed numerical procedure [7] that is able to accurately solve the full model in realistic parameter regimes. Where we used a combined asymptotic/numerical approach (solving for the ion vacancy distribution and electrical potential using the asymptotic model and solving for the charge carrier densities 
and currents numerically), we found extremely good agreement to the full numerical solution. In the case where we additionally linearised the charge carrier equations and solved them analytically, the comparison to the full numerical solution, while still good, was less impressive.

The physics of PSCs is still far from fully understood and in order to improve this situation, it is vital that drift-diffusion models and their solution techniques continue to be developed. One obvious, and important, extension to the model discussed here is the explicit inclusion of charge transport in the electron- and hole-transport layers on either side of the perovskite. Such an extension will be able to elucidate how the choice of these layers affects the cell's transient behaviours. In particular, this extended model could be used to investigate cell architectures giving rise to so-called low hysteresis behaviour and would also be better able to account for interfacial recombination, see, for example $[5,39]$. Here, we assume cation vacancies are immobile, which is justified by the relatively short timescales. However, it is believed that mobile methylammonium vacancies can lead to slow (over the timescale of many hours) but reversible changes in efficiency [9].

\section{Authors' contributions}

NEC and JMF coded the numerical solver and produced the plots. GR performed the asymptotic analysis. GR and NEC wrote the manuscript. ABW, SEJO'K and GR conceived the project and formulated the model.

\section{References}

[1] Advanpix (2017) Multiprecision Computing Toolbox for MATLAB version 4.3.2.12144.

[2] Black, J. P., Breward, C. J. \& Howell, P. D. (2017) Quantum mechanical effects in continuum charge flow models. IMA J. Appl. Math. 82, 251-279.

[3] Brinkman, D., Fellner, K., Markowich, P. A. \& Wolfram, M.-T. (2013) A drift-diffusionreaction model for excitonic photovoltaic bilayers: Asymptotic analysis and a 2D HDG finite element scheme. Math. Models Methods Appl. Sci. 23, 839-872.

[4] Brivio, F., Butler, K. T., Walsh, A. \& van SchilfGaArde, M. (2014) Relativistic quasiparticle self-consistent electronic structure of hybrid halide perovskite photovoltaic absorbers. Phys. Rev. B 89, 155204.

[5] Calado, P., Telford, A. M., Bryant, D., Li, X., Nelson, J., O’Regan, B. C. \& Barnes, P. R. (2016) Evidence for ion migration in hybrid perovskite solar cells with minimal hysteresis. Nat. Commun. 7, 13831.

[6] Correa-Baena, J.-P., Abate, A., Saliba, M., Tress, W., Jacobsson, T. J., Grätzel, M. \& Hagfeldt, A. (2017) The rapid evolution of highly efficient perovskite solar cells. Energy Environ. Sci. 10(3), 710-727.

[7] Courtier, N. E., Richardson, G. \& Foster, J. M. (2018) A fast and robust numerical scheme for solving models of charge carrier transport and ion vacancy motion in perovskite solar cells. arXiv:1801.05737v1.

[8] De Quilettes, D. W., Vorpahl, S. M., Stranks, S. D., Nagaoka, H., Eperon, G. E., Ziffer, M. E., SNaith, H. J. \& Ginger, D. S. (2015) Impact of microstructure on local carrier lifetime in perovskite solar cells. Science 348, 683-686.

[9] Domanski, K., Roose, B., Matsui, T., Saliba, M., Turren-Cruz, S.-H., Correa-Baena, J.-P., Carmona, C. R., Richardson, G., Foster, J. M., Angelis, F. D., Ball, J. M., Petrozza, A., Mine, N., Nazeeruddin, M. K., Tress, W., Grätzel, M., Steiner, U., Hagfeldt, A. \& 
AвAтE, A. (2017) Migration of cations induces reversible performance losses over day/night cycling in perovskite solar cells. Energy Environ. Sci. 10, 604-613.

[10] Eames, C., Frost, J. M., Barnes, P. R. F., O’Regan, B. C., Walsh, A. \& Islam, M. S. (2015) Ionic transport in hybrid lead iodide perovskite solar cells. Nat. Commun. 6, 7497.

[11] Foster, J. M., Kirk patrick, J. \& Richardson, G. (2013) Asymptotic and numerical prediction of current-voltage curves for an organic bilayer solar cell under varying illumination and comparison to the Shockley equivalent circuit. J. Appl. Phys. 114, 104501.

[12] Foster, J. M., Snaith, H. J., Leijtens, T. \& Richardson, G. (2014) A model for the operation of perovskite based hybrid solar cells: Formulation, analysis, and comparison to experiment. SIAM J. Appl. Math. 74, 1935-1966.

[13] Gottesman, R., Lopez-Varo, P., Gouda, L., Jimenez-Tejada, J. A., Hu, J., Tirosh, S., Zaban, A. \& BisquerT, J. (2016) Dynamic phenomena at perovskite/electron-selective contact interface as interpreted from photovoltage decays. Chem 1, 776-789.

[14] Kim, H.-S., Lee, C.-R., Im, J.-H., Lee, K.-B., Moehl, T., Marchioro, A., Moon, S.-J., HumphryBaker, R., Yum, J.-H., Moser, J. E., Grätzel, M. \& Park, N.-G. (2012) Lead iodide perovskite sensitized all-solid-state submicron thin film mesoscopic solar cell with efficiency exceeding 9\%. Sci. Rep. 2, 591.

[15] Kojima, A., Teshima, K., Shirai, Y. \& Miyasaka, T. (2009) Organometal halide perovskites as visible-light sensitizers for photovoltaic cells. J. Am. Chem. Soc. 131, 6050-6051.

[16] Koutselas, I. B., Ducasse, L. \& Papavassiliou, G. C. (1996) Electronic properties of threeand low-dimensional semiconducting materials with $\mathrm{Pb}$ halide and $\mathrm{Sn}$ halide units. J. Phys.: Condens. Matter 8, 1217-1227.

[17] Lee, M. M., Teuscher, J., Miyasaka, T., Murakami, T. N. \& Snaith, H. J. (2012) Efficient hybrid solar cells based on meso-superstructured organometal halide perovskites. Science 338, 643-647.

[18] Löper, P., Stuckelberger, M., Niesen, B., Werner, J., Filipič, M., Moon, S.-J., Yum, J.-H., Topič, M., Wolf, S. D. \& BALLIF, C. (2015) Complex refractive index spectra of $\mathrm{CH}_{3} \mathrm{NH}_{3} \mathrm{PbI}_{3}$ perovskite thin films determined by spectroscopic ellipsometry and spectrophotometry. $J$. Phys. Chem. Lett. 6(1), 66-71.

[19] The MathWorks, Inc. (2016) MATLAB version 9.1.0.441655 (R2016b).

[20] Nelson, J. (2003) The Physics of Solar Cells, Imperial College Press, London, UK.

[21] Neukom, M. T., Züfle, S., Knapp, E., Makha, M., Hany, R. \& Ruhstaller, B. (2017) Why perovskite solar cells with high efficiency show small IV-curve hysteresis. Sol. Energy Mater. Sol. Cells 169, 159-166.

[22] Niu, G., Guo, X. \& Wang, L. (2015) Review of recent progress in chemical stability of perovskite solar cells. J. Mater. Chem. A 3, 8970-8980.

[23] O’Kane, S. E. J., Richardson, G., Pockett, A., Niemann, R. G., Cave, J. M., Sakai, N., Eperon, G. E., Snaith, H. J., Foster, J. M., Cameron, P. J. \& Walker, A. B. (2017) Measurement and modelling of dark current decay transients in perovskite solar cells. J. Mater. Chem. C 5, 452-462.

[24] Please, C. (1982) An analysis of semiconductor P-N junctions. IMA J. Appl. Math. 28, 301-318.

[25] Pockett, A., Eperon, G. E., Peltola, T., Snaith, H. J., Walker, A., Peter, L. M. \& Cameron, P. J. (2015) Characterization of planar lead halide perovskite solar cells by impedance spectroscopy, open-circuit photovoltage decay, and intensity-modulated photovoltage/photocurrent spectroscopy. J. Phys. Chem. C 119, 3456-3465.

[26] Ravishankar, S., Almora, O., Echeverría-Arrondo, C., Ghahremanirad, E., Aranda, C., Guerrero, A., Fabregat-Santiago, F., Zaban, A., Garcia-Belmonte, G. \& Bisquert, J. (2017) Surface polarization model for the dynamic hysteresis of perovskite solar cells. $J$. Phys. Chem. Lett. 8, 915-921.

[27] Richardson, G. (2009) A multiscale approach to modelling electrochemical processes occurring across the cell membrane with application to transmission of action potentials. Math. Med. Biol. 26, 201-224. 
[28] Richardson, G., O’Kane, S. E. J., Niemann, R. G., Peltola, T. A., Foster, J. M., Cameron, P. J. \& WALKeR, A. B. (2016) Can slow-moving ions explain hysteresis in the current-voltage curves of perovskite solar cells? Energy Environ. Sci. 9, 1476-1485.

[29] Richardson, G., Please, C. \& Styles, V. (2017) Derivation and solution of effective medium equations for bulk heterojunction organic solar cells. Eur. J. Appl. Math. 28, 973-1014.

[30] Richardson, G. \& Walker, A. B. (2016) Drift diffusion modelling of charge transport in photovoltaic devices. In: E. Da Como, F. De Angelis, H. Snaith \& A. Walker (editors), Unconventional Thin Film Photovoltaics, Royal Society of Chemistry, Cambridge, UK, pp. 297-331.

[31] Schmeiser, C. (1992) Free boundaries in semiconductor devices. Proc. Free Boundary Problems: Theory and Applications. In: J. Chadham \& H. Rasmussen (editors), Pitman Research Notes Mathematics Series, vol. 3, Longman, Harlow, pp. 268-268.

[32] Schmeiser, C. \& Unterreiter, A. (1994) The derivation of analytic device models by asymptotic methods. In: W. M. Coughran, Jr., J. Cole, P. Lloyd \& J. K. White (editors), vol 59, Semiconductors. The IMA Volumes in Mathematics and its Applications, Springer, New York, NY, pp. 343-363.

[33] Schulz, P., Edri, E., Kirmayer, S., Hodes, G., Cahen, D. \& Kahn, A. (2014) Interface energetics in organo-metal halide perovskite-based photovoltaic cells. Energy Environ. Sci. 7, $1377-1381$.

[34] Shen, H., Jacobs, D. A., Wu, Y., Duong, T., Peng, J., Wen, X., Fu, X., Karuturi, S. K., White, T. P., Weber, K. \& Catchpole, K. R. (2017) Inverted hysteresis in $\mathrm{CH}_{3} \mathrm{NH}_{3} \mathrm{PbI}_{3}$ solar cells: Role of stoichiometry and band alignment. J. Phys. Chem. Lett. 8, 2672-2680.

[35] Snaith, H. J., Abate, A., Ball, J. M., Eperon, G. E., Leijtens, T., Noel, N. K., Stranks, S. D., Wang, J. T.-W., Wojciechowski, K. \& Zhang, W. (2014) Anomalous hysteresis in perovskite solar cells. J. Phys. Chem. Lett. 5, 1511-1515.

[36] Stoumpos, C. C., Malliakas, C. D. \& Kanatzidis, M. G. (2013) Semiconducting tin and lead iodide perovskites with organic cations: Phase transitions, high mobilities, and near-infrared photoluminescent properties. Inorg. Chem. 52, 9019-9038.

[37] Stranks, S. D., Burlakov, V. M., Leijtens, T., Ball, J. M., Goriely, A. \& Snaith, H. J. (2014) Recombination kinetics in organic-inorganic perovskites: Excitons, free charge, and subgap states. Phys. Rev. Appl. 2, 034007.

[38] Stranks, S. D. \& Snaith, H. J. (2015) Metal-halide perovskites for photovoltaic and lightemitting devices. Nat. Nanotechnol. 10, 391-402.

[39] Tan, H., Jain, A., Voznyy, O., Lan, X., de Arquer, F. P. G., Fan, J. Z., Quintero-BermudeZ, R., Yuan, M., Zhang, B., ZhaO, Y., Fan, F., Li, P., Quan, L. N., Zhao, Y., Lu, Z.-H., Yang, Z., Hoogland, S. \& Sargent, E. H. (2017) Efficient and stable solution-processed planar perovskite solar cells via contact passivation. Science 355, 722-726.

[40] van Reenen, S., Kemerink, M. \& Snaith, H. J. (2015) Modeling anomalous hysteresis in perovskite solar cells. J. Phys. Chem. Lett. 6, 3808-3814.

[41] Walsh, A., Scanlon, D. O., Chen, S., Gong, X. G. \& Wei, S.-H. (2015) Self-regulation mechanism for charged point defects in hybrid halide perovskites. Angew. Chem. 127, 18111814.

\section{Appendix Solution for $P$ and $\phi$ in the Debye layers}

In the bulk region, we obtain a solution for the leading order vacancy density $P_{0}^{(o)}$ and potential $\phi_{0}^{(o)}$, given by $P_{0}^{(o)}=1$ and $\phi_{0}^{(o)}=W_{-}(t)(1-x)+W_{+}(t) x$. These expressions satisfy the potential boundary conditions but in general cannot satisfy the flux boundary conditions, see (3.2). In order to resolve this seeming paradox, we need to account for narrow boundary layers (Debye layers) of width $\mathrm{O}(\lambda)$ about $x=0$ and $x=1$. 
Debye layer about $x=0$

Considering first the Debye layer about $x=0$, we use the rescaling (3.6) to rewrite the governing equations (3.1) and (3.2) in terms of the rescaled spatial variable $\zeta$, yielding the boundary layer equations:

$$
\begin{aligned}
\frac{\partial P}{\partial t} & +\frac{\partial \mathcal{F}_{p}}{\partial \zeta}=0, \quad \mathcal{F}_{p}=-\frac{1}{\lambda}\left(\frac{\partial P}{\partial \zeta}+P \frac{\partial \phi}{\partial \zeta}\right), \\
\frac{\partial^{2} \phi}{\partial \zeta^{2}} & =1-P, \\
\left.\phi\right|_{\zeta=0} & =\frac{\Phi-\Phi_{b i}}{2},\left.\quad \mathcal{F}_{p}\right|_{\zeta=0}=0,\left.\quad P\right|_{t=0}=1 .
\end{aligned}
$$

The expansions for $P, \phi$ and $\mathcal{F}_{p}$ proceed as in (3.7) so that to leading order in (1), we obtain the following equation for $P_{0}^{(d)}$ :

$$
\frac{\partial P_{0}^{(d)}}{\partial \zeta}+P_{0}^{(d)} \frac{\partial \phi_{0}^{(d)}}{\partial \zeta}=0
$$

This has the solution

$$
P_{0}^{(d)}=\exp \left(W(t)-\phi_{0}^{(d)}\right)
$$

for some as yet undetermined function of time, $W(t)$.

\section{Matching to the outer}

In order for the leading order Debye layer solution to match to the leading order outer solution, through (3.3) and (3.4), we require

$$
P_{0}^{(d)} \rightarrow 1, \quad \phi_{0}^{(d)} \rightarrow W_{-}(t), \quad \zeta \rightarrow+\infty
$$

Applying the matching condition (5) to the solution (4) determines a relation between the arbitrary functions $W(t)=W_{-}(t)$ motivating us to eliminate one of them by writing

$$
P_{0}^{(d)}=\exp \left(W_{-}(t)-\phi_{0}^{(d)}\right)
$$

On substituting this expression into (2) balanced at leading order, we find

$$
\frac{\partial^{2} \phi_{0}^{(d)}}{\partial \zeta^{2}}=1-\exp \left(W_{-}(t)-\phi_{0}^{(d)}\right)
$$

which satisfies boundary conditions obtained from the leading order terms in (3) and from (5), namely

$$
\left.\phi_{0}^{(d)}\right|_{\zeta=0}=\frac{\Phi_{b i}-\Phi}{2}, \quad \phi_{0}^{(d)} \rightarrow W_{-}(t), \zeta \rightarrow+\infty
$$

The corresponding expansion for the total charge per unit area in the left-hand Debye 
layer, $\mathcal{Q}_{-}($defined in (3.14)) is

$$
\mathcal{Q}_{-}=\mathcal{Q}_{-, 0}+\cdots
$$

and, by substituting this into (3.14), we obtain

$$
\mathcal{Q}_{-, 0}=\int_{0}^{\infty}\left(\exp \left(W(t)-\phi_{0}^{(d)}\right)-1\right) \mathrm{d} \zeta .
$$

We can reformulate the problem for $\phi_{0}^{(d)}$, given by (7) and (8), in a generic form by writing

$$
\phi_{0}^{(d)}(\zeta, t)=\theta\left(\zeta, \mathcal{V}_{-}(t)\right)+W_{-}(t)
$$

where $\mathcal{V}_{-}(t)$ represents the potential gained across the Debye layer, i.e., $\mathcal{V}_{-}=\left[\phi_{0}^{(d)}\right]_{0}^{\infty}$ (note that with this definition $\theta=-\log _{e} P_{0}^{(d)}$ ). It is then straightforward to show that the function $\theta(z, \mathcal{V})$ must satisfy the generic modified Poisson-Boltzmann problem

$$
\begin{aligned}
& \frac{\partial^{2} \theta}{\partial z^{2}}=1-e^{-\theta}, \\
& \left.\theta\right|_{z=0}=-\mathcal{V}_{-}(t), \quad \theta \rightarrow 0 \text { as } z \rightarrow+\infty
\end{aligned}
$$

Furthermore, in order that $\left.\phi_{0}^{(d)}\right|_{\zeta=0}=\frac{1}{2}\left(\Phi_{b i}-\Phi\right)$,

$$
W_{-}(t)=\frac{\Phi_{b i}-\Phi}{2}+\mathcal{V}_{-}(t)
$$

Thus, if we are able to determine $\mathcal{V}_{-}(t)$, we can determine the unknown function $W_{-}(t)$ in the leading order outer solution for the potential in (3.4).

It is straightforward to obtain a first integral to the autonomous equation (11) in the standard fashion by multiplying by $\theta_{z}$ and integrating with respect to $z$. This yields, on applying the far-field condition (12), the expression

$$
\frac{\partial \theta}{\partial z}=\operatorname{sign}(\mathcal{V}) \sqrt{2}\left(\theta+e^{-\theta}-1\right)^{1 / 2}
$$

where the $-\operatorname{sign}(\mathcal{V})$ is to account for the fact that if $\mathcal{V}<0(\mathcal{V}>0)$ the gradient of $\theta$ must be negative (positive). We can integrate (14) once more to obtain a relation for $z$ as a function of $\theta$ which reads

$$
\begin{aligned}
& z=\mathcal{Z}_{1}(\theta)-\mathcal{Z}_{1}(-\mathcal{V}) \quad \text { for } \quad \mathcal{V}>0 \quad \text { with } \quad \theta<0 \\
& z=\mathcal{Z}_{2}(\theta)-\mathcal{Z}_{2}(-\mathcal{V}) \text { for } \quad \mathcal{V}<0 \quad \text { with } \quad \theta>0
\end{aligned}
$$

where

$$
\begin{aligned}
& \mathcal{Z}_{1}(\theta)=\frac{1}{\sqrt{2}} \int_{-1}^{\theta} \frac{\mathrm{d} w}{\left(w+e^{-w}-1\right)^{1 / 2}} \quad \text { with } \quad \theta<0, \\
& \mathcal{Z}_{2}(\theta)=\frac{1}{\sqrt{2}} \int_{\theta}^{1} \frac{\mathrm{d} w}{\left(w+e^{-w}-1\right)^{1 / 2}} \quad \text { with } \quad \theta>0 .
\end{aligned}
$$


Characterising the capacitance of the Debye layer

We now seek to relate $\mathcal{Q}_{-, 0}$, as given in (9), to $\mathcal{V}_{-}$. We note that

$$
\mathcal{Q}_{-, 0}=\int_{0}^{\infty}\left(e^{-\theta}-1\right) \mathrm{d} \zeta
$$

which we can rewrite as

$$
\mathcal{Q}_{-, 0}=\int_{-\mathcal{V}_{-}}^{0} \frac{e^{-\theta}-1}{\theta_{\zeta}} \mathrm{d} \theta .
$$

On substituting for $\theta_{\zeta}$ from (14) and writing $\theta=-\mathcal{V}$ this integral transforms to

$$
\mathcal{Q}_{-, 0}=\frac{\operatorname{sign}\left(\mathcal{V}_{-}\right)}{\sqrt{2}} \int_{0}^{\mathcal{V}_{-}} \frac{e^{\mathcal{V}}-1}{\left(e^{\mathcal{V}}-\mathcal{V}-1\right)^{1 / 2}} \mathrm{~d} \mathcal{V}
$$

This integral can be further transformed by the substitution $M(\mathcal{V})=e^{\mathcal{V}}-\mathcal{V}-1$ to the exact integral

$$
\mathcal{Q}_{-, 0}=\frac{\operatorname{sign}\left(\mathcal{V}_{-}\right)}{\sqrt{2}} \int_{0}^{M\left(\mathcal{V}_{-}\right)} \frac{1}{M^{1 / 2}} \mathrm{~d} M,
$$

which yields the following exact relation between $\mathcal{Q}_{-, 0}$ and $\mathcal{V}_{-}$:

$$
\mathcal{Q}_{-, 0}=\operatorname{sign}\left(\mathcal{V}_{-}\right)\left(2\left(e^{\mathcal{V}_{-}}-\mathcal{V}_{-}-1\right)\right)^{1 / 2}
$$

This relation is plotted in Figure 3, from which it can be seen that $\mathcal{Q}_{-, 0}$ is a single valued function of $\mathcal{V}_{-}$. Hence, given the Debye layer charge density, $\mathcal{Q}_{-, 0}$, we can invert to find the potential jump across the Debye layer, $\mathcal{V}_{-}$. This motivates us to consider the evolution of $\mathcal{Q}_{-, 0}(t)$ as charge (in the form of positively charged vacancies) flow out into (or in from) the bulk region.

\section{A solvability condition on $\mathcal{Q}_{-, 0}(t)$}

It remains to determine the evolution of $\mathcal{V}_{-}(t)$. This can be done by tracking the charge build up in the Debye layer through the leading order expansion of the positively charged vacancy conservation equation (1),

$$
\frac{\partial P_{0}^{(d)}}{\partial t}+\frac{\partial \mathcal{F}_{p, 0}^{(d)}}{\partial \zeta}=0
$$

and the boundary conditions

$$
\left.\mathcal{F}_{p, 0}^{(d)}\right|_{\zeta=0}=0,\left.\quad \mathcal{F}_{p, 0}^{(d)} \rightarrow \mathcal{F}_{p, 0}^{(o)}\right|_{x=0} \text { as } \zeta \rightarrow+\infty .
$$

These conditions are obtained from the leading order expansion of $(3 b)$ and from matching to the leading order outer solution as $\zeta \rightarrow+\infty$, respectively. By writing $\partial P_{0}^{(d)} / \partial t$ as $(\partial / \partial t)\left(1-P_{0}^{(d)}\right)$, integrating $(18)$ between $\zeta=0$ and $\zeta=\infty$ and applying the flux 
boundary conditions (19), we obtain the solvability condition

$$
\frac{d \mathcal{Q}_{-, 0}}{d t}=-\left.\mathcal{F}_{p, 0}^{(o)}\right|_{x=0}
$$

\section{The Debye layer about $x=1$}

The analysis of this right-hand layer proceeds in a similar fashion to the left-hand Debye layer presented above. We introduce the rescaled spatial variable $\xi$, defined in (3.10), and then expand as follows:

$$
\begin{aligned}
P & =P_{0}^{(D)}(\xi, t)+\cdots, & & \mathcal{F}_{p}=\mathcal{F}_{p, 0}^{(D)}(\xi, t)+\cdots, \\
\phi & =\phi_{0}^{(D)}(\xi, t)+\cdots, & & \mathcal{Q}_{+}=\mathcal{Q}_{+, 0}(t)+\cdots .
\end{aligned}
$$

Following an analogous series of steps to the analysis of the left-hand layer, we find that

$$
P_{0}^{(D)}=\exp \left(W_{+}(t)-\phi_{0}^{(D)}\right)
$$

and that the leading order potential satisfies the problem

$$
\begin{aligned}
& \frac{\partial^{2} \phi_{0}^{(D)}}{\partial \xi^{2}}=1-\exp \left(W_{+}(t)-\phi_{0}^{(D)}\right), \\
& \left.\phi_{0}^{(D)}\right|_{\xi=0}=-\frac{\Phi_{b i}-\Phi}{2} \\
& \phi_{0}^{(D)} \rightarrow W_{+}(t) \text { as } \xi \rightarrow \infty .
\end{aligned}
$$

The solution to this problem is very similar to that for $\phi_{0}^{(d)}(\zeta, t)$ being given by

$$
\phi_{0}^{(D)}(\xi, t)=\theta\left(\xi, \mathcal{V}_{+}(t)\right)+W_{+}(t)
$$

where $\mathcal{V}_{+}(t)=\left[\phi_{0}^{(D)}\right]_{\xi=0}^{\infty}$ is the jump in potential across the right-hand Debye layer and the function $\theta(\cdot, \cdot)$ is (as before) a solution to (11) and (12); in other words, one can make the transformation $\zeta \rightarrow \xi$ and $\mathcal{V}_{-} \rightarrow \mathcal{V}_{+}$in $\theta\left(\zeta, \mathcal{V}_{-}\right)$both here and in the implicit solution for $\theta$ given in (15). In addition, it follows from the condition that $\left.\phi_{0}^{(D)}\right|_{\xi=0}=-\frac{1}{2}\left(\Phi_{b i}-\Phi\right)$ that

$$
W_{+}(t)=-\frac{\Phi_{b i}-\Phi}{2}+\mathcal{V}_{+}(t)
$$

In a similar manner to that described above, we determine a relation between $\mathcal{Q}_{+, 0}$ and $\mathcal{V}_{+}$which is identical to (17) and reads

$$
\mathcal{Q}_{+, 0}=\operatorname{sign}\left(\mathcal{V}_{+}\right)\left(2\left(e^{\mathcal{V}_{+}}-\mathcal{V}_{+}-1\right)\right)^{1 / 2}
$$

Once again, a solvability condition may be derived from the problem for the leading order anion vacancy density, namely,

$$
\frac{\partial P_{0}^{(D)}}{\partial t}-\frac{\partial \mathcal{F}_{p, 0}^{(D)}}{\partial \xi}=0,
$$




$$
\left.\mathcal{F}_{p, 0}^{(D)}\right|_{\xi=0}=0,\left.\quad \mathcal{F}_{p, 0}^{(D)} \rightarrow \mathcal{F}_{p, 0}^{(o)}\right|_{x=1} \text { as } \xi \rightarrow+\infty .
$$

The solvability condition we obtain on integrating this system is the following evolution equation for $\mathcal{Q}_{+, 0}(t)$ :

$$
\frac{d \mathcal{Q}_{+, 0}}{d t}=\left.\mathcal{F}_{p, 0}^{(o)}\right|_{x=1}
$$

